\title{
Decode-and-Forward Cooperation-Aided Triple-Layer Turbo-Trellis-Coded Hierarchical Modulation
}

\author{
Hua Sun, Soon Xin Ng, Senior Member, IEEE, Chen Dong, and Lajos Hanzo, Fellow, IEEE
}

\begin{abstract}
Hierarchical modulation (HM) is widely employed across the telecommunication industry. The potential application of the coded HM scheme in cooperative communications has drawn much interest. In this paper, a twin-relay-aided triple-layer cooperative communication system is proposed. The system amalgamates rate-1/2 TTCM, triple-layer HM-64QAM, and twin-layer SPM-16QAM schemes in the context of cooperative communications. We have optimized the entire system based on the HM ratio pair $\left(R_{1}, R_{2}\right)$, the superposition modulation (SPM) weighting pair $(\alpha, \beta)$, and the positions of the two relays. The simulation results show that our optimized system is capable of reliably transmitting a triple-layer HM-64QAM signal with the aid of two time slots at an average signal-to-noise ratio of $6.94 \mathrm{~dB}$ per time slot.
\end{abstract}

Index Terms-Hierarchical modulation, superposition modulation, turbo trellis-coded modulation, cooperative communication, soft decoding and power efficiency.

\section{INTRODUCTION}

$\mathbf{H}$ IERARCHICAL modulation (HM) constitutes an integral part of the DVB-T/-H standard [1], but it has also been widely employed by the IT industry for upgrading diverse telecommunication services [2], [3]. Compared to a system using conventional modulation, the system employing HM has a higher flexibility, while maintaining backward compatibility. Explicitly, both the original and the upgraded new services may be combined by the HM scheme and broadcast to the receivers without requiring any additional bandwidth. Although the services is upgraded to a higher data rate, the original devices are still supported by the upgraded broadcast system without requiring software or hardware upgrade [4].

HM has been developed for combining independent information streams at bit-level layer by layer, which are then mapped onto HM constellations. The information contained in different

Manuscript received July 9, 2014; revised November 5, 2014; accepted December 19, 2014. This work was supported in part by the European Research Council under the Advanced Fellowship Grant, by the EPSRC through IndiaU.K. Advanced Technology Centre, and by the EU's Concerto Project. The associate editor coordinating the review of this paper and approving it for publication was G. Bauch.

The authors are with the School of Electronics and Computer Science, University of Southampton, Southampton SO17 1BJ, U.K.

Color versions of one or more of the figures in this paper are available online at http://ieeexplore.ieee.org.

Digital Object Identifier 10.1109/TCOMM.2014.2387164 layers may also be demapped/detected separately. The general performance of the HM scheme have been detailed in [5]-[8]. It can be observed from the simulation results of [5], [6] that different layers in HM constellations receive different protection levels. Consequently, the required received Signal-to-Noise Ratio $\left(\mathrm{SNR}_{r}\right)$ for receiving the layer with higher protection level requires lower $\mathrm{SNR}_{r}$ than that of the less protected layers. The author of [9] employed HM in his system to provide unequal error protection (UEP) for the information contained in different layers. It has drawn a lot of interests [10]-[15]. More specifically, the authors of [13], [14] invoked a HM scheme for providing UEP for video and image encoding, where the information bits are mapped to specific protection layers according to their error-sensitivity-based priority. Moreover, the HM scheme has also been combined with sophisticated channel coding schemes in [13], [14], for protecting the most important information. The simulation results of [13], [14] have shown that receiving the information having the highest priority requires a lower $\mathrm{SNR}_{r}$ compared to conventional modulation schemes at a given target BER performance.

A typical relay aided coded HM scheme was introduced in [16], where Hausl and Hagenauer combined Turbo Coding (TC) [17] with a HM scheme conceived for cooperative communications, where the original signal sequence was broadcast by the Source Node (SN) by ensuring that the layer with higher protection may be received by the Destination Node (DN) directly, while the less protected layer will be received and retransmitted by the Relay Node (RN). However, the authors of [16] only considered the specific scenario, when the position of the RN is right in the middle of the SN-DN path and invoked a specific bit-to-symbol mapping scheme. The performance of the coded HM schemes was then further discussed in [18][21] in the context of cooperative communications, where the common choice is to employ multiple encoders at the $\mathrm{SN}$ and combine all the coded bit sequences layer-by-layer to create a HM signal sequence. Again, the less well-protected layers are assisted by the RN of the cooperative network.

The bit-to-symbol mapping optimization of the HM scheme was considered in [20], [22]. More specifically, by appropriately designing the constellation mapping, the HM scheme is capable of enhancing the protection of the higher-priority information at the expense of providing a weaker protection for the other layers. In [21], the specific position of the $\mathrm{RN}$ was explicitly considered in the BER analysis. For a specific coded $\mathrm{HM}$ scheme aided system, the receive power at the RN should 
be sufficiently high for guaranteeing that the RN becomes capable of receiving the information in the lower protected layer with an acceptable integrity. Therefore, the position of the RN may influence the power allocation of the entire system. Several parameters have to be taken into consideration, when optimizing a coded HM aided cooperative communication system. On one hand, distorting the HM constellation for the sake of improving the BER of its high-priority layers at the detriment of its low-priority layers degrades its average BER, compared to conventional modulation schemes. On the other hand, sophisticated channel coding schemes, such as TrellisCoded Modulation (TCM), Turbo Trellis-Coded Modulation (TTCM) and Bit-Interleaved Coded Modulation (BICM) [17], [23]-[25] are required for protecting each HM layer at the expense of an increased complexity. Hence, giving cognizance both to the complexity and to the power efficiency of the overall system, while maintaining its flexibility becomes a challenging task.

In [26], a cooperative communication system assisted by a TTCM based HM-16QAM scheme was proposed, where we proposed the optimum constellation mapping for the HM-16QAM scheme in the context of single RN aided cooperative communications. However, the position of the RN was fixed to be right in the middle of the SN-DN path. Hence, the scheme of [26] was suboptimal, because its power allocation was suboptimal. Against this background, in this treatise we intrinsically amalgamate HM, Superposition Modulation (SPM) [27] and TTCM for creating an attractive cooperative communication system. Our goal is to increase the time-efficiency and reduce the total power consumption of the entire system, while maintaining a low complexity. This cooperative communication system model may be readily used for assisting multilayer video transmission for example [13], [28] or for multilayer image transmission [14]. We exploit the idealized simplifying assumption that the system benefits from perfect Channel State Information (CSI), including both the fading and path-loss. Hence, according to the receiver's SNR, we are capable of determining the transmission power required at the transmitter, which we defined as the transmit SNR $\left(\mathrm{SNR}_{t}\right)^{1}$. We proposed a Turbo Trellis-Coded Hierarchical Modulation (TTCHM) aided twin-relay based cooperative communication scheme, where three rate-1/2 TTCM encoders are employed at the $\mathrm{SN}$ for constructing a 64QAM-based triple-layer HM scheme. The TTCM scheme detailed in [17] has a better performance for transmission over Rayleigh fading channels than other joint coding and modulation schemes, such as TCM and BICM. An excellent performance can be attained by TTCM without expanding the bandwidth. A rate-k/(k+1) TTCM scheme can be used for protecting a k-bit HM layer by expanding the number of constellation points from $2^{k}$ to $2^{k+1}$. We considered $k=1$ in this contribution.

Depending on the specific symbol-to-bit demapping arrangement of the HM scheme, different HM layers have different

\footnotetext{
${ }^{1}$ The definition of transmit SNR was proposed in [29], which is convenient for simplifying the discussions, although this is not a physically measurable quantity, because it relates the power at the transmitter to the noise at the receivers.
}

protections. Explicitly, the information in the higher-protection layers may require a lower $\mathrm{SNR}_{r}$ at the $\mathrm{DN}$ than that of the information in the lower-protection layers. Hence, when the SN transmits a multi-layer $\mathrm{HM}$ signal in our cooperative communication network, the $\mathrm{SNR}_{t}$ at the $\mathrm{SN}\left(\mathrm{SNR}_{t}^{S N}\right)$ may be reduced to the minimum value that can 'just' guarantee the successful detection of the base layer (highest priority) of the $\mathrm{HM}$ signal at the DN. By contrast, the information in the lower priority layers may be received and retransmitted by the RN. Since we proposed a triple-layer HM scheme, two RNs are activated for retransmitting the information of the two lower layers. More specifically, a linear SPM scheme is employed by the two RNs for simultaneously transmitting the two signal frames to the DN during the second time slot (TS). Hence, two TSs are required for the transmission of all the three layers from the SN to the DN. Since the transmissions between the SN and $\mathrm{RN}$ (or RN and DN) only deal with a single 4QAM layer of the triple-layer HM-64QAM signals, the decoding complexity imposed on the two RNs (and DN) is reduced. Moreover, the $\mathrm{SNR}_{t}$ at the $\mathrm{RN}\left(\mathrm{SNR}_{t}^{R N}\right)$ can also be minimized, because both RNs will only retransmit using 4QAM. If only one RN is available for assisting the transmissions, the $\mathrm{RN}$ would have to detect both enhancement layers from the HM-64QAM signals. The position of this RN would be near to the $\mathrm{SN}$ and the transmission between the RN and the $\mathrm{DN}$ will be based on 16QAM modulation. This would require a high $\mathrm{SNR}_{t}$ due to the transmission of a higher-order modulation scheme over a longer distance. Due to the flexibility of HM, the lowestprotection layer that contains the least important information can be discarded in the adverse situation, when none of the RNs is capable of detecting it. Nonetheless, the DN can still receive the pair of more important layers of the HM-64QAM. We found that apart from reducing the power dissipation of the entire system, the processing complexity of the twin-relay aided cooperative communication network may also be mitigated, when an appropriate design is invoked.

The main contributions of this paper are as follows:

- A triple-layer HM-64QAM scheme is designed for aiding a Decode-and-Forward (DF) based cooperative communications, which involves intrinsically amalgamating TTCM, HM and SPM schemes;

- Based on our Monte-Carlo simulations, a power-allocation is conceived and it is demonstrated that the power consumption of the entire system may be readily optimized by relying on the related variables, namely by the HM-based symbol-energy ratio pair $\left(R_{1}, R_{2}\right)$, by the SPM weighting pair $(\alpha, \beta)$, and by the relay's geographic position.

The rest of the paper is organized as follows. Section II introduces both the system model and our cooperative communication strategy. The specific HM-64QAM mapping rule designed for cooperative communication is detailed in Section III. The protocol of the symbol-to-bit demapper of the HM symbols is discussed in Section IV. The triple-layer TTCHM-64QAM system design is detailed in Section V and the simulation results are displayed in Section VI, our conclusions and future research ideas are discussed in Section VII. 


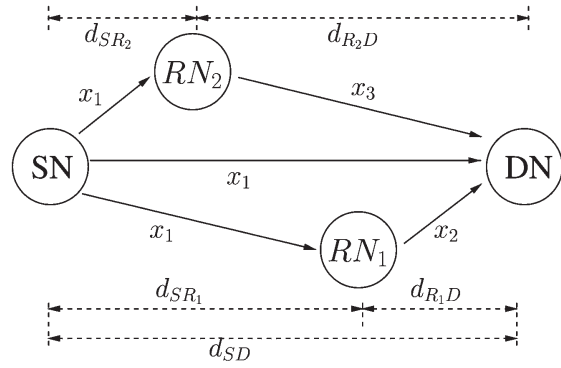

Fig. 1. The model of a two-relay cooperative system.

\section{System Model}

Our TTCHM aided DF RN based cooperative communication system is illustrated in Fig. 1. During the first transmission TS, the $\mathrm{SN}$ will broadcast a sequence of TTCHM symbols $\left\{x_{1}\right\}$ to $\mathrm{RN}_{1}, \mathrm{RN}_{2}$ and $\mathrm{DN}$. In the following TS, $\mathrm{RN}_{1}$ will transmit a signal frame $\left\{x_{2}\right\}$ to the $\mathrm{DN}$, another signal frame $\left\{x_{3}\right\}$ will also be sent to the $\mathrm{DN}$ by the $\mathrm{RN}_{2}$ simultaneously. Again, the entire system would require two TSs to convey the triple-layer TTCHM-64QAM symbol based signal frame $\left\{x_{1}\right\}$ to DN.

We considered an uncorrelated Rayleigh flat-fading channel, both the transmitters and receivers were assumed to acquire perfect CSI. During the first TS, each symbol received by the DN may be expressed as:

$$
y_{S D}=\sqrt{G_{S D}} h_{S D} x_{1}+n_{S D},
$$

where each of the symbols received by the $\mathrm{RN}_{1}$ and $\mathrm{RN}_{2}$ are:

$$
\begin{aligned}
& y_{S R_{1}}=\sqrt{G_{S R_{1}}} h_{S R_{1}} x_{1}+n_{S R_{1}}, \\
& y_{S R_{2}}=\sqrt{G_{S R_{2}}} h_{S R_{2}} x_{1}+n_{S R_{2}},
\end{aligned}
$$

where the subscript SD denotes the SN-DN link and the subscript $\mathrm{SR}_{k}$ represents the $\mathrm{SN}-\mathrm{RN}_{k}$ link. By contrast, the symbols received at the DN during the second TS, which are sent by the two RNs, may be expressed as:

$$
y_{R D}=\rho_{1} \alpha \sqrt{G_{R_{1} D}} h_{R_{1} D} x_{2}+\rho_{2} \beta \sqrt{G_{R_{2} D}} h_{R_{2} D} x_{3}+n_{R D},
$$

where $\rho_{1}$ and $\rho_{2}$ are the pre-coding parameters and $(\alpha, \beta)$ is the SPM ratio pair. The subscript $\mathrm{R}_{k} \mathrm{D}$ represents the $\mathrm{RN}_{k}-\mathrm{DN}$ link. Additionally, the notations $h_{S D}, h_{S R_{k}}$ and $h_{R_{k} D}$ denote the complex-valued coefficients of the uncorrelated Rayleigh fading for the different links, $n_{S D}, n_{S R_{k}}$ and $n_{R_{k} D}$ denote the Additive White Gaussian Noise (AWGN) having a variance of $N_{0} / 2$ per dimension. Moreover, the variables $G_{S D}, G_{S R_{k}}$ and $G_{R_{k} D}$ represent the Reduced-Distance-Related-PathlossReduction (RDRPLR) for each link, which we also refer to as the path-gain [25], [29]-[31]. We consider an inverse-power law based non-free-space path-loss model and naturally and we define the path-loss exponent to be 3 which is usually used to simulate the path-loss in urban areas [32]. The path-gain $G_{S D}$ of the SD link is assumed to be unity. Therefore the path-gains of the two SR links are:

$$
G_{S R_{k}}=\left(\frac{d_{S D}}{d_{S R_{k}}}\right)^{3}
$$

and similarly, the path-gains of the two RD links are:

$$
G_{R_{k} D}=\left(\frac{d_{S D}}{d_{R_{k} D}}\right)^{3}
$$

We assume that every node in the cooperative network has perfect CSI. Hence, given a specific path-loss and a RN position, we may both compensate the effect of the path-loss as well as that of the Rayleigh fading with the aid of transmit pre-coding. Specifically, the $\rho_{1}$ and $\rho_{2}$ pre-coding parameters should satisfy:

$$
\begin{aligned}
& \rho_{1}=\frac{h_{R_{1} D}^{*}}{\left|h_{R_{1} D}\right|^{2} \sqrt{G_{R_{1} D}}}, \\
& \rho_{2}=\frac{h_{R_{2} D}^{*}}{\left|h_{R_{2} D}\right|^{2} \sqrt{G_{R_{2} D}}} .
\end{aligned}
$$

Hence, during the second TS, the signal received by the DN may be written as:

$$
y_{R D}=\alpha x_{2}+\beta x_{3}+n_{R D} .
$$

In a realistic situation, there is always a path-loss between the SN and DN, but in order to simplify the system model, we normalized this path-loss to $0 \mathrm{~dB}$. Hence the transmit power at the $\mathrm{SN}\left(\mathrm{SNR}_{t}^{S N}\right)$ would be identical to the power received at the $\mathrm{DN}\left(\mathrm{SNR}_{r}^{D N}\right)$. If the transmissions between the $\mathrm{SN}$ and DN are on a frame-by-frame basis over an uncorrelated Rayleigh fast fading channel, the average received SNR at DN $\left(\overline{S N R_{r}^{D N}}\right)$ would be given by:

$$
\overline{S N R_{r}^{D N}}=E\left(|h|^{2} S N R_{t}\right)=E\left(|h|^{2}\right) S N R_{t}^{S N},
$$

where the $\mathrm{SNR}_{t}^{S N}$ is the transmit SNR defined as the ratio of the transmit power to the noise power at the DN:

$$
S N R_{t}^{S N}=\frac{E\left(|x|^{2}\right)}{N_{0}}=\frac{1}{N_{0}},
$$

where $E\left(|x|^{2}\right)=1$. Furthermore, the uncorrelated Rayleigh fading coefficient $h$ is generated by the complex-valued Gaussian distribution having a zero mean and a variance of one. When the number of uncorrelated Rayleigh fading coefficients we generated is large, we have [33]:

$$
E\left(|h|^{2}\right)=\frac{1}{N} \sum_{k=1}^{N}\left|h_{k}\right|^{2} \approx 1 .
$$

Hence, for a large frame size of $N$ symbols, we may assume that $\mathrm{SNR}_{r}^{D N}$ is equal to the $\mathrm{SNR}_{t}^{S N}$, or equivalently $\overline{S N R_{r}^{D N}}=S N R_{t}^{S N}$.

To be more specific, the information flow of the entire system is illustrated in the block diagram shown in Fig. 2. In our system, the SN employs three rate-1/2 4QAM-TTCM encoders and combines the three independent codeword sequences into a HM signal stream. Thus, the signal frame $\left\{x_{1}\right\}$ is formed by HM-64QAM symbols. When the transmit power at the source is relatively low, the DN may opt for decoding only the information from Encoder 1 during the first TS, where the 


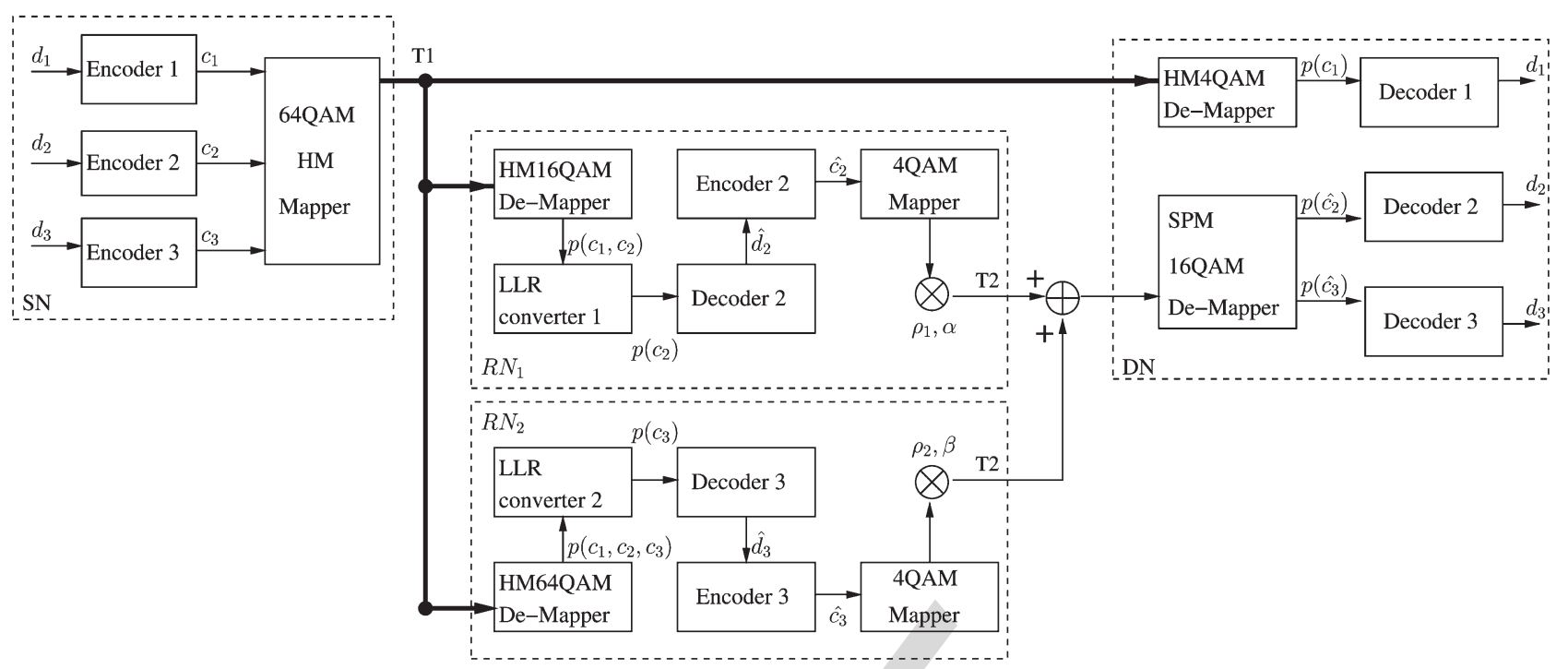

Fig. 2. The system diagram of a twin-relay HM and SPM aided cooperative system.

information contained in the other two layers would be decoded and retransmitted by the RNs. In order to reduce the complexity of the entire system, two RNs are activated for assisting the transmissions and each of the two RNs is used for retransmitting only one information layer of the triple-layer HM-64QAM symbols. With aid of the pre-coding and SPM schemes, the two RNs become capable of transmitting simultaneously. Hence, the system now needs two TSs to complete its transmissions between the SN and DN. More specifically, each RN will only deal with a single layer of the triple-layer HM-64QAM scheme, so the signal frames they transmit are all 4QAM symbol frames. In this way, both the processing complexity of the entire system and the transmit power of the $\mathrm{RN}$ is reduced.

\section{HM AND SPM MODULATION SCHEMES}

\section{A. Triple Layer HM Scheme}

Our triple-layer model of the HM-64QAM constellation seen in Fig. 3 was originally introduced in [26]. Since TTCM is used, where the symbol-based decoder's performance is determined by the Symbol Error Rate (SER), hence set-partition based mapping is invoked by the HM constellation instead of Gray mapping.

We define the six bits in a HM-64QAM symbol as $\left(b_{5} b_{4} b_{3} b_{2} b_{1} b_{0}\right)$, where the base layer or first layer $\left(L_{1}\right)$ is occupied by $\left(b_{5} b_{4}\right),\left(b_{3} b_{2}\right)$ belong to the second layer $\left(L_{2}\right)$ and $\left(b_{1} b_{0}\right)$ are contained in the third layer $\left(L_{3}\right)$. The generation rule of the triple-layer HM-64QAM symbols may be expressed as:

$$
S_{H M-64 Q A M}=\beta\left[S_{4 Q A M} \pm \sqrt{2} \delta_{1} e^{ \pm \frac{\pi}{4} j} \pm \sqrt{2} \delta_{2} e^{ \pm \frac{\pi}{4} j}\right] .
$$

The parameter $\beta$ is used for normalizing the average symbol energy to unity, which given by $\beta=1 / \sqrt{1+2 \delta_{1}^{2}+2 \delta_{2}^{2}}$. Furthermore, the ratios $R_{1}=d_{1} / d_{0}$ and $R_{2}=d_{3} / d_{2}$ are defined for controlling the shape of the HM-64QAM constellations, as shown in Fig. 3, where all the three parameters $\beta, \delta_{1}$ and $\delta_{2}$ will

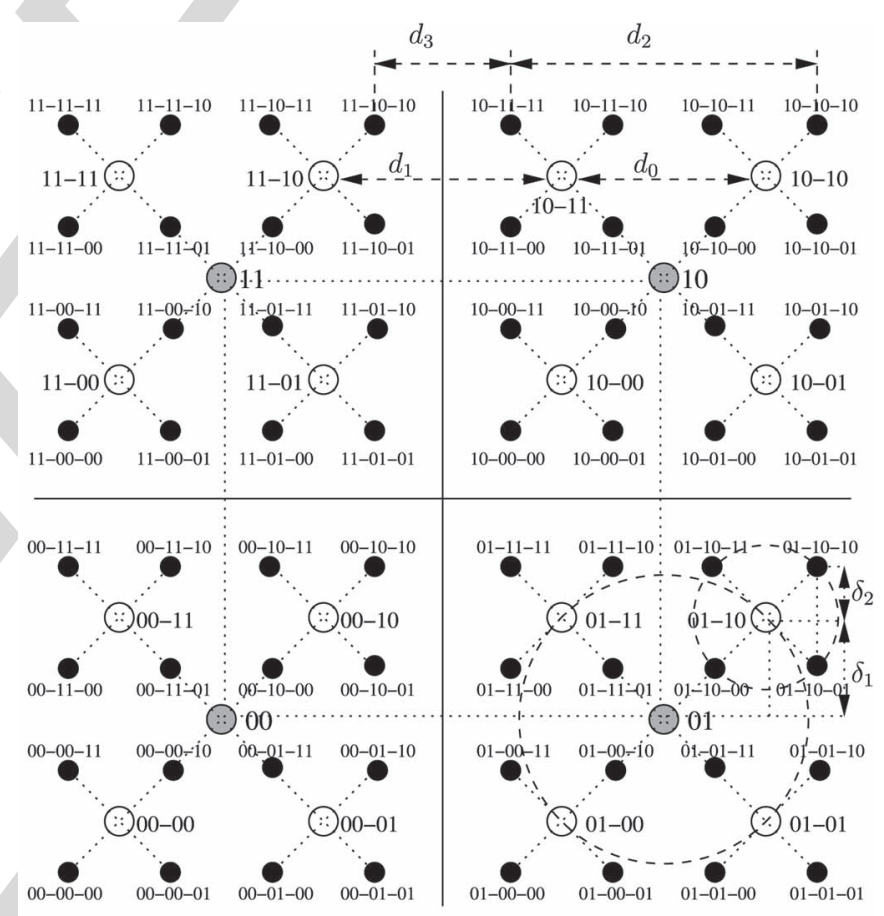

Base Layer: $b_{5} b_{4}$

HM 16QAM: $b_{5} b_{4}-b_{3} b_{2}$

HM 64QAM: $b_{5} b_{4}-b_{3} b_{2}-b_{1} b_{0}$

Fig. 3. The constellation map of the triple-layer HM-64QAM scheme, where $R_{1}=d_{1} / d_{0}, R_{2}=d_{3} / d_{2}$.

be directly controlled by the HM ratio pair $\left(R_{1}, R_{2}\right)$ and their relationship may be expressed as follows:

$$
\begin{aligned}
\delta_{1} & =\frac{1}{\sqrt{2}\left(1+R_{1}\right)}, \\
\delta_{2} & =\frac{R_{1}-R_{2}}{\sqrt{2}\left(1+R_{1}\right)\left(1+R_{2}\right)} .
\end{aligned}
$$


The constraint of the HM ratio pair $\left(R_{1}, R_{2}\right)$ in the simulations are:

$$
\begin{cases}0<R_{2}<R_{1} & \text { if } R_{1}<1 \\ \frac{1}{2}\left(R_{1}-1\right)<R_{2}<R_{1} & \text { if } R_{1}>1\end{cases}
$$

The derivations of (14), (15) and (16) are detailed in Appendices A, B, C and D respectively. The entire HM-64QAM constellation point arrangement is directly controlled by the HM ratios $R_{1}$ and $R_{2}$. Upon increasing the value of these two HM ratios, the constellation points in each quadrant would move closer to each other. Hence it is necessary to have a higher receive $\mathrm{SNR}$ at the $\mathrm{RN}\left(\mathrm{SNR}_{r}^{R N}\right)$ in order to adequately detect the information contained both in $L_{2}$ as well as in $L_{3}$, but a lower $\mathrm{SNR}_{r}^{D N}$ is necessitated for detecting the two bits in $L_{1}$. Our design-goal is to find the optimum $\mathrm{HM}$ ratios and RN position based on a given $\mathrm{SNR}_{t}^{S N}$.

\section{B. Twin Layer SPM Scheme}

The twin layer SPM scheme is detailed in [34], where we observe from this (9) that the reception of the signal at the DN is identical to that of detecting a twin-layer linear SPM signal received over AWGN channels. Note that the performance of a specific modulation scheme in the AWGN channel is directly determined by the Euclidean distance among the constellation points. The relationship between $\alpha$ and $\beta$ is given by [34]:

$$
\alpha^{2}+\beta^{2}=1
$$

If we only focus our attention on the relationship between the Euclidean distance $d_{\min }$ and $\alpha$, we have:

$d_{\min }=\left\{\begin{array}{ll}\frac{1}{\sqrt{2}} \min \left(2 \sqrt{1-\alpha^{2}}, 2\left(\alpha-\sqrt{1-\alpha^{2}}\right)\right) & (\alpha \geq \sqrt{1 / 2}) \\ \frac{1}{\sqrt{2}} \min \left(2 \alpha, 2\left(\sqrt{1-\alpha^{2}}-\alpha\right)\right) & (\alpha<\sqrt{1 / 2})\end{array}\right.$.

Theoretically the largest Euclidean distance is achieved, when $\alpha$ is $\sqrt{1 / 5}$ or $\sqrt{4 / 5}$. Hence, we anticipate that the best performance of an uncoded twin-layer linear SPM scheme would appear when $\alpha$ equals to $\sqrt{1 / 5}$ or $\sqrt{4 / 5}$. However, when TTCM is used, it depends on $d_{\min }$ of the entire TTCM set partitioning scheme [17].

\section{DEMAPPER AND RN POSITION}

In [34], we have discussed the receiving of the two signal frames using SPM schemes, so in this section, we only focus our attention on the receiving of the triple-layer TTCHM64QAM symbols. The symbol-to-bit Demapper block of Fig. 2 will produce a $(N \times M)$-element Probability Density Function (PDF) matrix of receiving $y$ given $x^{(i)}$ transmitted. $x^{(i)}$ is the hypothetically transmitted $M$-ray symbol for $i \in$ $\{0,1, \cdots, M-1\}$ and the element in the matrix is $p\left(y \mid x^{(i)}\right)$, which is the soft-input to the TTCM decoder, $N$ is the number of symbols in a transmission block. The general equation of calculating the PDF of receiving $y$, given that $x^{(i)}$ is transmitted may be expressed as:

$$
p\left(y \mid x^{(i)}\right)=\frac{1}{\pi N_{0}} \exp \left(\begin{array}{r}
\left.-\frac{\left|y-\sqrt{G} h x^{(i)}\right|^{2}}{N_{0}}\right) \\
\quad \times i \in\{0,1, \ldots, M-1\},
\end{array}\right.
$$

where $h$ is the fading coefficient and $G$ is the path-gain.

\section{A. $L_{1}$ Detection at $D N$}

The DN of Fig. 2 will demap the HM-64QAM signal frames received from the SN as 4QAM symbols for detecting the information contained in the base layer of the triple-layer HM-64QAM constellation. According to the HM-64QAM generation rule of (13), (19) may be rewritten as:

$$
\begin{gathered}
p\left(y_{S D} \mid x^{(i)}\right)=\frac{1}{\pi N_{0}} \exp \left(-\frac{\left|y_{S D}-\sqrt{G_{S D}} h_{S D} x^{(i)}\right|^{2}}{N_{0}}\right) \\
\times x^{(i)} \in\left\{\beta e^{j \pi / 4}, \beta e^{j 3 \pi / 4}, \beta e^{j-3 \pi / 4}, \beta e^{j-\pi / 4}\right\},
\end{gathered}
$$

where we have $i \in\{0,1,2,3\}$.

\section{B. $L_{2}$ Detection at $R N_{1}$}

The information output of Encoder 2 in Fig. 2 is mapped onto $L_{2}, R N_{1}$ will demap the received signal frame $x_{1}$ as the HM-16QAM symbols shown in Fig. 3 and will obtain the joint symbol probability of $L_{1}$ and $L_{2}$ in the HM-64QAM symbol streams for producing a $(N \times 16)$-element PDF matrix. Then, the Log Likelihood Ratio (LLR) converter 1 of Fig. 2, will extract the PDF of $L_{2}$ from the $(N \times 16)$-element PDF matrix. Therefore, $R N_{1}$ can decode $L_{2}$ even when $L_{1}$ is received with errors. When demapping the HM-64QAM symbol as HM-16QAM, (19) may be reformulated as:

$$
\begin{aligned}
p\left(y_{S R_{1}} \mid x^{(i)}\right)= & \frac{1}{\pi N_{0}} \exp \left(-\frac{\left|y_{S R_{1}}-\sqrt{G_{S R_{1}}} h_{S R_{1}} x^{(i)}\right|^{2}}{N_{0}}\right) \\
& \times x^{(i)} \in\left\{\beta\left[S_{4 Q A M} \pm \sqrt{2} \delta_{1} e^{ \pm \frac{\pi}{4} j}\right]\right\}, \quad(21)
\end{aligned}
$$

where $i \in\{0,1, \cdots, 15\}$. The HM-16QAM constellation points of $x^{(i)}$ are shown as hollow circles in Fig. 3. We defined $L_{2}^{(0)}$ as the pair of bits (00) in $L_{2}, L_{2}^{(1)}$ as $(01), L_{2}^{(2)}(10)$ and finally $L_{2}^{(3)}$ for (11), where the corresponding generation rule is given by:

$$
\begin{aligned}
p\left(y_{S R_{1}} \mid L_{2}^{(l)}\right) & =p\left(y_{S R_{1}} \mid x^{(l)}\right)+p\left(y_{S R_{1}} \mid x^{(l+4)}\right) \\
& +p\left(y_{S R_{1}} \mid x^{(l+8)}\right)+p\left(y_{S R_{1}} \mid x^{(l+12)}\right),
\end{aligned}
$$

where $l \in\{0,1,2,3\}$. The input PDF matrix of Decoder 2 is formulated in (22).

\section{C. $L_{3}$ Detection at $R N_{2}$}

$R N_{2}$ requires the highest receive power, because it has to convey the information of $L_{3}$. In order to receive $L_{3}, R N_{2}$ has 
to fully demap the whole HM-64QAM symbol stream. Hence (19) may be represented as:

$$
\begin{gathered}
p\left(y_{S R_{2}} \mid x^{(i)}\right)=\frac{1}{\pi N_{0}} \exp \left(-\frac{\left|y_{S R_{2}}-\sqrt{G_{S R_{2}}} h_{S R_{2}} x^{(i)}\right|^{2}}{N_{0}}\right) \\
\times x^{(i)} \in\left\{\beta\left[S_{4 Q A M} \pm \sqrt{2} \delta_{1} e^{ \pm \frac{\pi}{4} j} \pm \sqrt{2} \delta_{2} e^{ \pm \frac{\pi}{4} j}\right]\right\},
\end{gathered}
$$

where $i \in\{0,1, \cdots, 63\}$. Let $L_{3}^{(0)}$ denote the pair of bits (00) in $L_{3}, L_{3}^{(1)}$ represent (01), $L_{2}^{(3)}(10)$ and finally $L_{3}^{(3)}$ for (11). Then the LLR converter 2 of Fig. 2 may produce the PDF of $L_{3}$ according to:

$$
p\left(y_{S R_{2}} \mid L_{3}^{(l)}\right)=\sum_{k=0}^{15} p\left(y_{S R_{1}} \mid x^{(i=4 k+l)}\right),
$$

where $l \in\{0,1,2,3\}$. The $(N \times 4)$-element PDF matrix generated by the LLR converter 2 is then fed to Decoder 3 of $\mathrm{RN}_{2}$, as seen in Fig. 2, for detecting $L_{3}$. Furthermore, we consider logarithmic probabilities, so that the approximate log MAP algorithm [17] may be directly employed by the decoder block.

\section{RN Position}

In our simulations, the same rate- $1 / 2$ encoder is employed for all three SN encoders. Hence we only focus our attention on the specific SNR values for achieving a BER of $10^{-6}$. Multiple values of the two HM ratios $R_{1}$ and $R_{2}$ had been tested. At a given $\mathrm{HM}$ constellation ratio pair $\left(R_{1}, R_{2}\right)$, the minimum receive SNR required $S N R_{r}^{L_{1}}$ for decoding $L_{1}$ at the DN, $S N R_{r}^{L_{2}}$ for receive $L_{2}$ at the $R N_{1}$ and $S N R_{r}^{L_{3}}$ for receiving $L_{3}$ at $R N_{2}$ may be computed. The SNR differences among the three layers are:

$$
\begin{aligned}
& \mathcal{G}_{S N R}^{L_{1}, L_{2}}=S N R_{r}^{L_{2}}-S N R_{r}^{L_{1}}[\mathrm{~dB}], \\
& \mathcal{G}_{S N R}^{L_{1}, L_{3}}=S N R_{r}^{L_{3}}-S N R_{r}^{L_{1}}[\mathrm{~dB}],
\end{aligned}
$$

where, $\mathcal{G}_{S N R}^{L_{1}, L_{j}}$ is the SNR difference between $S N R_{r}^{L_{1}}$ and $S N R_{r}^{L_{j}}$, for $j \in\{2,3\}$. If we set $\operatorname{SNR}_{t}^{S N}$ to be identical to the SNR required for receiving $L_{1}$ from the HM-64QAM symbol, namely to $S N R_{t}^{S N}=S N R_{r}^{L_{1}}$, this would guarantee that the BER of decoding $L_{1}$ would reach an arbitrarily low value. In this situation, if we want the BER performance of receiving $L_{2}$ to become sufficiently low, the channel gain $G_{S R_{1}}$ of the $\mathrm{SN}-\mathrm{RN}_{1}$ link should satisfy:

$$
10 \log _{10} G_{S R_{1}}+S N R_{r}^{L_{1}}=S N R_{r}^{L_{2}} .
$$

If we use the distance-ratio $d_{S R_{1}} / d_{S D}$ to represent the position of the RN, we arrive at:

$$
\mathcal{G}_{S N R}^{L_{1}, L_{2}}=10 \log _{10}\left(\frac{d_{S D}}{d_{S R_{1}}}\right)^{3}
$$

where $\mathcal{G}_{S N R}^{L_{1}, L_{2}}$ is given by (25) and hence we have:

$$
\frac{d_{S R_{1}}}{d_{S D}}=10^{-\frac{\mathcal{G}_{S N R}^{L_{1} L_{2}}}{30}} .
$$

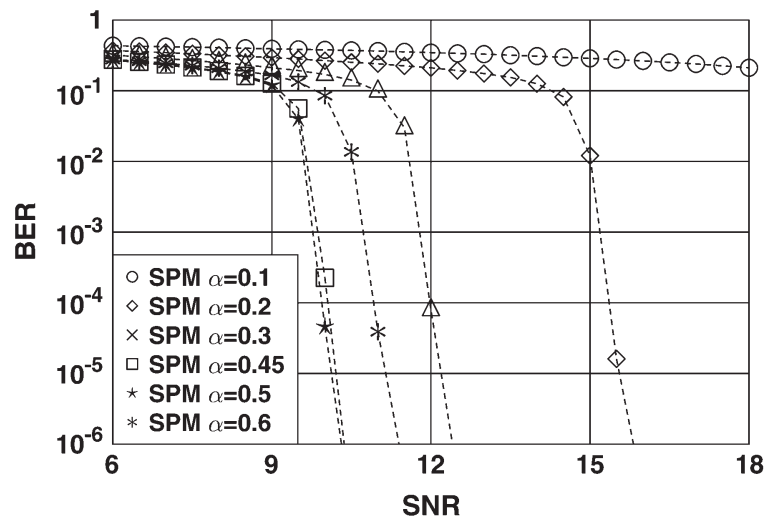

(a)

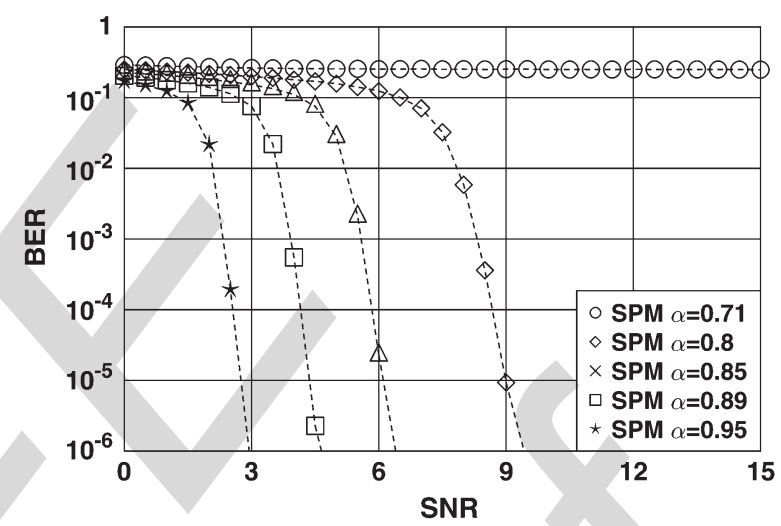

(b)

Fig. 4. The BER versus SNR performance of receiving a single signal sequence with SPM ratio $\alpha$ in the twin layer SPM schemes. Both of the two signal sequences are encoded by rate-1/2 TTCM encoder. The number of iterations of the rate-1/2 TTCM decoder is $\zeta=4$, the block size is $\eta=12,000$ symbols and the channel is uncorrelated Rayleigh fading channel.

Similarly, the position of $\mathrm{RN}_{2}$ is related to:

$$
\frac{d_{S R_{2}}}{d_{S D}}=10^{-\frac{\mathcal{G}_{S N R}^{L_{1}, L_{3}}}{30}} .
$$

\section{TRIPLE-LAYER TTCHM-64QAM CoOPERATIVE SYSTEM DESIGN}

In practice we do not have any control over the position of mobile relays, but the relay-selection algorithm would appoint a relay close to the optimum location. In this section, we will optimize this practical system regarding the position of the RN, as well as the HM ratio pair $\left(R_{1}, R_{2}\right)$ and the SPM weighting pair $(\alpha, \beta)$. Additionally, in this investigation, the simulations are carried out by IT++and the number of iterations of our rate-1/2 TTCM decoder is $\zeta=4$, the block size is $\eta=12,000$ symbols. Using a large number of iterations allows the TTCM decoder to more closely approach capacity and a large block length assists in avoiding error propagation, but also imposes an increased complexity. In the simulations, we observed that no substantial BER performance improvement is achieved for more than four iterations $(\zeta>4)$ or for a block size of $\eta>$ 12,000 symbols. 


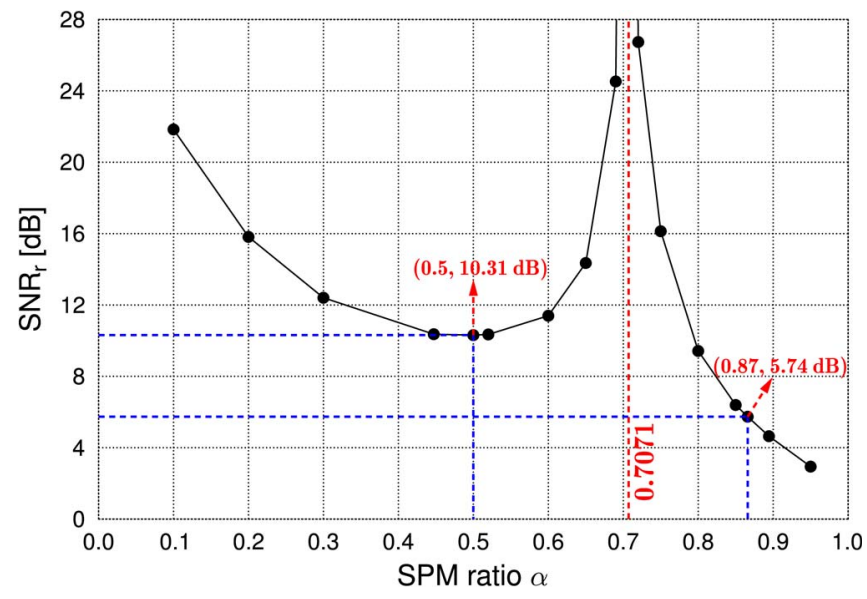

Fig. 5. The $\mathrm{SNR}_{r}$ versus SPM ratio performance of receiving a single signal sequence with SPM ratio $\alpha$ in the twin layer SPM schemes. Both of the two signal sequences are encoded by rate- $1 / 2$ TTCM encoder. The number of iterations of the rate- $1 / 2$ TTCM decoder is $\zeta=4$, the block size is $\eta=$ 12,000 symbols and the channel is uncorrelated Rayleigh fading channel. The $\mathrm{SNR}_{r}$ here is the required receive $\mathrm{SNR} r$ for achieving a performance of $\mathrm{BER}=10^{-6}$.

\section{A. Optimum SPM Ratio}

In our simulations, the pair of signal sequences received from $\mathrm{RN}_{1}$ and $\mathrm{RN}_{2}$ will be multiplied by a specific SPM ratio $\alpha$ or $\beta$ and be received by the DN simultaneously. Here, we only focus our attention on the performance of receiving a single signal sequence having a SPM ratio $\alpha$ in the simulations and the related results are shown in Fig. 4(a) and Fig. 4(b).

From the combined signal sequences, we set the signal associated with a larger SPM ratio $\alpha$ to be the dominant signal and the signal with a smaller SPM ratio $\beta$ to be the auxiliary signal. Based on the simulation results of Fig. 4(a) and Fig. 4(b), we may observe that as expected, for the dominant signal, a larger SPM ratio would result in a better BER performance. However, for the auxiliary signal, the best BER performance appears at the SPM ratio of $0.45(\sqrt{1 / 5} \approx 0.45)$ or 0.5 . We have found from our simulations the $\mathrm{SNR}_{r}$ value required for achieving $\mathrm{BER}=10^{-6}$ for different values of the SPM ratio $\alpha$, as shown in Fig. 5. Note that $0.7071(\sqrt{1 / 2} \approx 0.7071)$ is a bound, where we have $\alpha=\beta=\sqrt{1 / 2}$, which would collapse the 16 -point constellation to a 9-point constellation making unambiguous decoding to be impossible. Fig. 5 shows that when $\alpha=0.5$, the $\mathrm{SNR}_{r}$ required for receiving the auxiliary signal from the SPM16QAM signal is about $10.31 \mathrm{~dB}$ which is the lowest value of receiving the auxiliary signal. Meanwhile, when $\alpha=0.5$, the corresponding SPM ratio $\beta$ will be $\sqrt{1-\alpha^{2}} \approx 0.87$, and the $\mathrm{SNR}_{r}$ required for receiving the dominant signal from the SPM16QAM signal using $\beta=0.87$ is about $5.74 \mathrm{~dB}$. Hence, based on the results of Fig. 5, we find that the optimum SPM ratio pair is ( $\alpha=0.87, \beta=0.5)$ (we assume $\alpha>\beta$ in this paper). In this combination, the $\mathrm{SNR}_{r}$ required for receiving the two signal sequences is the lowest. Consequently, the $\mathrm{SNR}_{t}$ required at the two RNs would also be the lowest at the given RN positions.

\section{B. SNR of the Two RNs}

In order to evaluate the power-efficiency of our cooperative communications scheme, we have to calculate the average
$\mathrm{SNR}_{t}\left(\overline{S N R_{t}}\right)$ defined as the $\mathrm{SNR}_{t}$ per TS. Based on the statistics seen in Fig. 5, we opted for the SPM ratio pair of ( $\alpha=$ $0.87, \beta=0.5)$ and hence we have $\operatorname{SNR}_{r}^{D N}=10.31 \mathrm{~dB}$. Next, we have to find the relationship among $\mathrm{SNR}_{t}^{R N_{1}}, \mathrm{SNR}_{t}^{R N_{2}}$ and $\operatorname{SNR}_{r}^{D N}$. Let us denote the SNR by $\gamma$, which is expressed as $10 \log _{10}(\gamma)$ in $\mathrm{dB}$. Furthermore, since $L_{2}$ of the triple-layer HM-64QAM symbols has a higher priority than that of $L_{3}$, the signal frame $\left\{x_{2}\right\}$ transmitted from $\mathrm{RN}_{1}$ will be multiplied by a higher SPM weighting factor of $\alpha=0.87$, while another SPM weighting factor of $\beta=0.5$ is used for the signal frame $\left\{x_{3}\right\}$ at $\mathrm{RN}_{2}$. Hence we have:

$$
E\left[\gamma_{t}^{R N_{1}}\right]=E\left[\frac{\left|\alpha \rho_{1} x_{2}\right|^{2}}{N_{0}}\right]
$$

where

$$
E\left[\left|x_{2}\right|^{2}\right]=1
$$

Therefore, it can be observed that:

$$
\begin{aligned}
E\left[\gamma_{t}^{R N_{1}}\right] & =E\left[\frac{\alpha^{2}\left|\rho_{1}\right|^{2}}{N_{0}}\right] \\
& =\alpha^{2} E\left[\frac{\left|h_{R_{1} D}^{*}\right|^{2}}{\left|h_{R_{1} D}\right|^{4} G_{R_{1} D} N_{0}}\right] \\
& =\frac{\alpha^{2}}{G_{R_{1} D} N_{0}} E\left[\frac{1}{\left|h_{R_{1} D}\right|^{2}}\right],
\end{aligned}
$$

where $h$ obeys the Rayleigh distribution of [32]:

$$
f(h)=\frac{2 h}{\Omega} \exp \left(-\frac{h^{2}}{\Omega}\right) .
$$

Note that the mean square value of $h$ is given by $\Omega=1$, Let $Z=|h|^{2}$, then the distribution of the variable $Z$ may be expressed as [32]:

$$
f_{Z}(z)=\frac{1}{\Omega} \exp \left(-\frac{z}{\Omega}\right) .
$$

Let us denote the Cumulative Distribution Function (CDF) as $F_{Z}(\Gamma)=\operatorname{Pr}(Z<\Gamma)$, which can be expressed as:

$$
F_{Z}(\Gamma)=\operatorname{Pr}(Z<\Gamma)=1-\exp \left(-\frac{\Gamma}{\Omega}\right),
$$

where $\operatorname{Pr}$ denotes probability. Upon introducing $\Gamma^{\prime}=1 / \Gamma$, we may further express (36) as:

$$
\operatorname{Pr}(Z<\Gamma)=\operatorname{Pr}\left(\frac{1}{Z}>\frac{1}{\Gamma}\right)=1-\operatorname{Pr}\left(\frac{1}{Z}<\Gamma^{\prime}\right) .
$$

Hence, it can be observed that:

$$
\operatorname{Pr}\left(\frac{1}{Z}<\Gamma^{\prime}\right)=\exp \left(-\frac{1}{\Omega \Gamma^{\prime}}\right) \text {. }
$$

If we let $\Theta=\frac{1}{Z}$, the PDF of the variable $\Theta$ may be expressed as [35]:

$$
f_{\Theta}(\theta)=\frac{1}{\Omega \theta^{2}} \exp \left(-\frac{1}{\Omega \theta}\right)
$$


Therefore, the expectation $E\left[\frac{1}{|h|^{2}}\right]$ may be derived as:

$$
E\left[\frac{1}{|h|^{2}}\right]=\int_{\Theta_{\text {low }}}^{\Theta_{u p}} \frac{\Theta}{\Omega \Theta^{2}} \exp \left(-\frac{1}{\Omega \Theta}\right) \mathrm{d} \Theta,
$$

where $\Theta_{\text {low }}$ and $\Theta_{\text {up }}$ are the lower and upper limits of the integration. Let us define $\xi=\frac{1}{\Theta}$, then (40) may be converted to:

$$
\frac{1}{\Omega} \int_{\frac{1}{\Theta_{u p}}}^{\frac{1}{\Theta_{\text {low }}}} \frac{1}{\xi} \exp \left(-\frac{\xi}{\Omega}\right) \mathrm{d} \xi=\frac{1}{\Omega}\left[E_{i}\left(\frac{-1}{\Omega \Theta_{\text {up }}}\right)-E_{i}\left(\frac{-1}{\Omega \Theta_{\text {low }}}\right)\right] \text {, }
$$

where $E_{i}$ is the Euler function:

$$
E_{i}(u)=\int_{-u}^{\infty} \frac{e^{-t}}{t} \mathrm{~d} t
$$

Theoretically, we have $|h| \in[0,+\infty)$, which gives us $\Theta_{\text {low }}=0$ and $\Theta_{\text {up }}=+\infty$. When $\Theta_{\text {low }}=0$, we may have $E_{i}\left(\frac{-1}{\Omega \Theta_{\text {low }}}\right)=0$. However, if $\Theta_{u p}=+\infty$, the term $E_{i}\left(\frac{-1}{\Omega \Theta_{u p}}\right)$ becomes infinite and we are unable to derive the value of $E\left[1 /|h|^{2}\right]$. To resolve this dilemma, we defined an outage threshold, which is given by $\left[|h|^{2}\right]_{\min }=0.03$. According to (35), the probability of $\operatorname{Pr}\left(|h|^{2} \geqslant 0.03\right) \approx 0.97$, indicates that our system will halt its transmissions, when the fading obeys $|h|^{2}<0.03$. Hence, we may have $\frac{1}{\Theta_{u p}}=\left[|h|^{2}\right]_{\min }=0.03$. In this situation, we have $E\left[1 /|h|^{2}\right]=2.96$. Hence, (33) may be expressed as:

$$
E\left[\gamma_{t}^{R N_{1}}\right]=\frac{2.96 \alpha^{2}}{G_{R_{1} D} N_{0}}
$$

Note that this assumption will lead to a $3 \%$ throughput reduction for the entire system. Additionally, we have:

$$
E\left[\gamma_{r}^{D N}\right]=E\left[\frac{\left|\alpha x_{2}+\beta x_{3}\right|^{2}}{N_{0}}\right]=\frac{1}{N_{0}} .
$$

Therefore, we find that:

$$
E\left[\gamma_{t}^{R N_{1}}\right]=\frac{2.96 \alpha^{2}}{G_{R_{1} D}} E\left[\gamma_{r}^{D N}\right]
$$

which may be expressed in $\mathrm{dB}$ as:

$$
E\left[S N R_{t}^{R N_{1}}\right]=E\left[S N R_{r}^{D N}\right]+10 \log _{10}\left(\frac{2.96 \alpha^{2}}{G_{R_{1} D}}\right) .
$$

Similarly, the relationship between $S N R_{t}^{R N_{2}}$ and $S N R_{r}^{D N}$ may be formulated as:

$$
E\left[S N R_{t}^{R N_{2}}\right]=E\left[S N R_{r}^{D N}\right]+10 \log _{10}\left(\frac{2.96 \beta^{2}}{G_{R_{2} D}}\right) .
$$

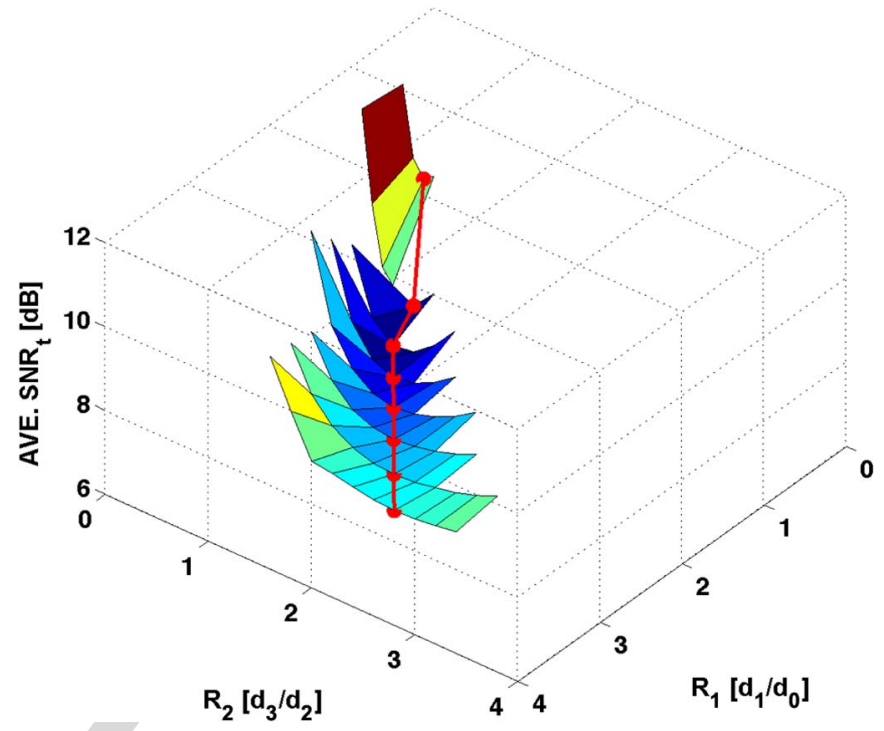

Fig. 6. The 3D plot of the simulation-based transmit power dissipation surface of the entire system versus the HM-64QAM ratio pair $\left(R_{1}, R_{2}\right)$. The two RNs employ SPM schemes associated with the SPM weighting pair of $(\alpha=0.87$, $\beta=0.5$ ). Explicitly, the number of iterations of the rate-1/2 TTCM decoder is $\zeta=4$, the block size is $\eta=12,000$ symbols and an uncorrelated Rayleigh fading channel is considered.

Finally, $\overline{S N R_{t}}$ may be expressed as:

$\overline{S N R_{t}}=10 \log _{10}\left(\overline{\gamma_{t}}\right)=10 \log _{10}\left(\frac{\gamma_{t}^{S N}+\gamma_{t}^{R N_{1}}+\gamma_{t}^{R N_{2}}}{2}\right)$.

\section{Simulation Results}

Based on Section V, we characterized our cooperative communications system for multiple values of the HM ratio pairs $\left(R_{1}, R_{2}\right)$ for the sake of generating the power dissipation surface of the cooperative system, which is shown in Fig. 6. Explicitly, 64 pairs of $\left(R_{1}, R_{2}\right)$ have been simulated, where $R_{1}$ is chosen from $\{0.5,1.0,1.5,2.0,2.5,3.0,3.5,4.0\}$ and for each $R_{1}$, multiple $R_{2}$ values were selected based on the constraints illustrated in Section III. Given a specific HM ratio pair $\left(R_{1}, R_{2}\right)$, the receive SNR required for adequately receiving $L_{1}, L_{2}$ and $L_{3}$, namely $\mathrm{SNR}_{r}^{L_{1}}, \mathrm{SNR}_{r}^{L_{2}}$ and $\mathrm{SNR}_{r}^{L_{3}}$ respectively, may be derived for a target BER of $10^{-6}$. More specifically, the optimum position of the two RNs and the optimum average transmit $\operatorname{SNR}\left(\overline{S N R_{t}}\right)$ of the entire system may be calculated according to $\mathrm{SNR}_{r}^{L_{1}}, \mathrm{SNR}_{r}^{L_{2}}$ and $\mathrm{SNR}_{r}^{L_{3}}$ based on the discussions in Sections IV and Section V. Hence, we can compute the optimum $\overline{S N R_{t}}$ for each of the HM ratio pairs $\left(R_{1}, R_{2}\right)$, as shown in Fig. 6 . The bold line marked by dots in Fig. 6 illustrates the lowest power consumption point for a specific $\mathrm{HM}$ ratio pair $\left(R_{1}, R_{2}\right)$ and the corresponding data is recorded in Table I.

Based on the results of Fig. 6, the best performance of our cooperative communication system obeying this arrangement is achieved, when the HM ratio pair is given by $\left(R_{1}=1.5\right.$, $R_{2}=0.8$ ) and the SPM weighting factor pair is ( $\alpha=0.87, \beta=$ 0.5 ), where the optimum $\overline{S N R_{t}}$ per TS is $6.94 \mathrm{~dB}$. In this situation, the positions of $\mathrm{RN}_{1}$ and $\mathrm{RN}_{2}$ are $d_{S R_{1}} / d_{S D}=0.53$ and $d_{S R_{2}} / d_{S D}=0.31$, where $\mathrm{SNR}_{t}^{S N}$ is $6.81 \mathrm{~dB}, \mathrm{SNR}_{t}^{R N_{1}}$ is 
TABLE I

The Simulation Based Average Transmission Power $\overline{S N R_{t}}$ Per TS of the HM-64QAM and SPM Based Cooperative System. The SPM WeIghting PAIR IS $(\alpha=0.87, \beta=0.5)$, THE NUMBER OF ITERATIONS OF THE RATE- $1 / 2$ TTCM DECODER IS $\zeta=4$, AND THE BLOCK SizE

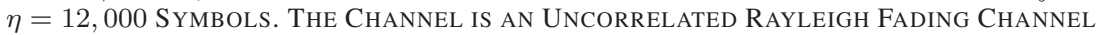

\begin{tabular}{|l|l|l|l|l|l|l|l|}
\hline$R_{1}$ & $R_{2}$ & $\begin{array}{l}\text { Pos. } \\
{\left[d_{S R_{1}} / d_{S D}\right]}\end{array}$ & $\begin{array}{l}\text { Pos. } \\
{\left[d_{S R_{2}} / d_{S D}\right]}\end{array}$ & $\begin{array}{l}S N R_{t}^{S N} \\
{[\mathrm{~dB}]}\end{array}$ & $\begin{array}{l}S N R_{t}^{R N_{1}} \\
{[\mathrm{~dB}]}\end{array}$ & $\begin{array}{l}S N R_{t}^{R N_{2}} \\
{[\mathrm{~dB}]}\end{array}$ & $\begin{array}{l}S N R_{t} \\
{[\mathrm{~dB}]}\end{array}$ \\
\hline 0.5 & 0.3 & 0.82 & 0.33 & 11.18 & -8.81 & 3.88 & 8.94 \\
\hline 1.0 & 0.6 & 0.65 & 0.30 & 7.98 & -0.04 & 4.27 & 6.96 \\
\hline 1.5 & 0.8 & 0.53 & 0.31 & 6.81 & 4.01 & 4.08 & $\mathbf{6 . 9 4}$ \\
\hline 2.0 & 1.2 & 0.47 & 0.26 & 5.91 & 5.61 & 5.12 & 7.32 \\
\hline 2.5 & 1.6 & 0.42 & 0.22 & 5.42 & 6.71 & 5.76 & 7.76 \\
\hline 3.0 & 2.0 & 0.38 & 0.19 & 5.06 & 7.52 & 6.20 & 8.14 \\
\hline 3.5 & 2.4 & 0.35 & 0.17 & 4.79 & 8.10 & 6.50 & 8.46 \\
\hline 4.0 & 2.8 & 0.33 & 0.16 & & 6.73 & 8.74 \\
\hline
\end{tabular}

$4.01 \mathrm{~dB}$ and $\mathrm{SNR}_{t}^{R N_{2}}$ is $4.08 \mathrm{~dB}$, as shown in Table I. The throughput per TS for this scheme is $3 / 2 \times 0.97=1.455 \mathrm{bps}$ owing to the $3 \%$ throughput reduction imposed by the threshold of $\left[|h|^{2}\right]_{\min }=0.03$.

\section{A. EXIT Chart Analysis}

The Extrinsic Information Transfer (EXIT) chart is capable of visualizing the input/output characteristics of the constituent MAP decoders in terms of the achievable average mutual information transfer [36], [37]. It may be used for predicting whether a soft decision based decoder is capable of decoding the information with an arbitrarily low BER based on the available mutual information provided by the symbol-to-bit demapper. Since we do not invoke iterations between the demapper and the TTCM decoder in our symbol-based scheme, we need a sufficiently high receive $\mathrm{SNR}_{r}$ for guaranteeing that the mutual information gleaned from the demapper is sufficiently high for the decoder to attain a low BER. More specifically, the EXIT curves of the HM-64QAM and SPM-16QAM demappers are illustrated in Fig. 7.

In Fig. 7, the notation 'Inner' iteration represents the information exchange between the demapper and decoder, while 'Outer' iteration refers to the information exchange between the two components of the TTCM decoder. Since there are no iterations between the demapper and the TTCM decoder, we may observe in Fig. 7 that the inner curve is a straight line. Specifically, the inner curve shows the mutual information received by the decoder from the demapper, which is increased upon increasing the receive SNR. In order to guarantee that the decoder becomes capable of decoding the information with an arbitrarily low BER, an open EXIT chart tunnel has to be maintained between the 'Inner' and 'Outer' curves all the way to the $(x, y)=(2, y)$ point, where we have $x=I_{A}^{(i)}=I_{E}^{(o)}$ and $y=I_{E}^{(i)}=I_{A}^{(o)}$. Note that the subscript $i$ and $o$ denote 'Inner' and 'Outer' respectively, whilst $I_{A}$ and $I_{E}$ denote the a priori and extrinsic information. When the HM-64QAM ratio pair is ( $R_{1}=1.5, R_{2}=0.8$ ) and the number of TTCM iterations is $\zeta=4$, the receive SNR required for achieving a BER of $10^{-6}$ for each HM layer is: $\mathrm{SNR}_{r}^{L_{1}}=6.81 \mathrm{~dB}, \mathrm{SNR}_{r}^{L_{2}}=15.15 \mathrm{~dB}$ and $\mathrm{SNR}_{r}^{L_{3}}=21.87 \mathrm{~dB}$. It also can be observed in Fig. 7 that there are open tunnels between the three 'Inner' curves upon receiving triple-layer HM symbols and the $\zeta=4$-iteration 'Outer' curve. Note that the 'Inner' curve of receiving $L_{3}$ of the

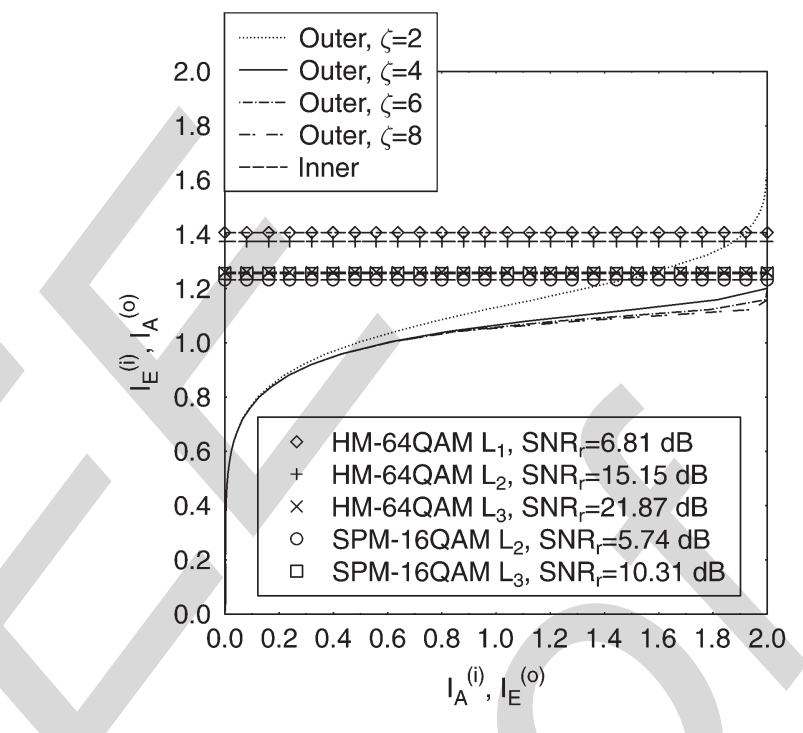

Fig. 7. The symbol based EXIT chart of our rate-1/2 TTCM aided triplelayer HM-64QAM scheme and twin-layer SPM-16QAM scheme. The number of iterations in the rate-1/2 TTCM decoder is from the set of $\zeta \in\{2,4,6,8\}$. The HM-64QAM ratio pair is $\left(R_{1}=1.5, R_{2}=0.8\right)$ and the SPM weighting coefficient pair is $(\alpha=0.87, \beta=0.5)$. The receive SNR required for achieving a BER of $10^{-6}$ based on simulations is denoted as $\mathrm{SNR}_{r}$. An uncorrelated Rayleigh fading channel is considered.

HM scheme is closer to the 'Outer' curve compared to the other two 'Inner' curves of receiving $L_{1}$ and $L_{2}$. When $L_{1}$ of the HM scheme is detected at the DN, each HM-64QAM symbol received will be detected as a 4QAM symbol. Similarly, $L_{2}$ of the HM scheme will be detected in the form of 16QAM symbols at $\mathrm{RN}_{1}$. This HM-specific simplifying demapping assumption shifts the 'Inner' curves corresponding to $L_{1}$ and $L_{2}$ upwards to higher values than the 'Inner' curve recorded for receiving $L_{3}$. Hence, we infer that we can reduce the SNR required for receiving $L_{1}$ and $L_{2}$ to let the two 'Inner' curves to be closer to the $\zeta=4$-iteration based 'Outer' curve. However, according to the simulations, even though there might be an open EXIT tunnel, the integrity of $L_{1}$ and $L_{2}$ will be degraded, if we reduce $\mathrm{SNR}_{r}$.

Additionally, the 'Inner' curves associated with receiving the pre-coding based twin-layer SPM-16QAM scheme are also shown in Fig. 7. In the simulations, the $L_{2}$ and $L_{3}$ of the triplelayer HM-64QAM will be mapped to base layer and auxiliary layer of the SPM symbols, respectively. It can be observed in Fig. 7 that the $\mathrm{SNR}_{r}$ required for achieving a BER of $10^{-6}$ for both layers of our twin-layer SPM scheme can provide open 


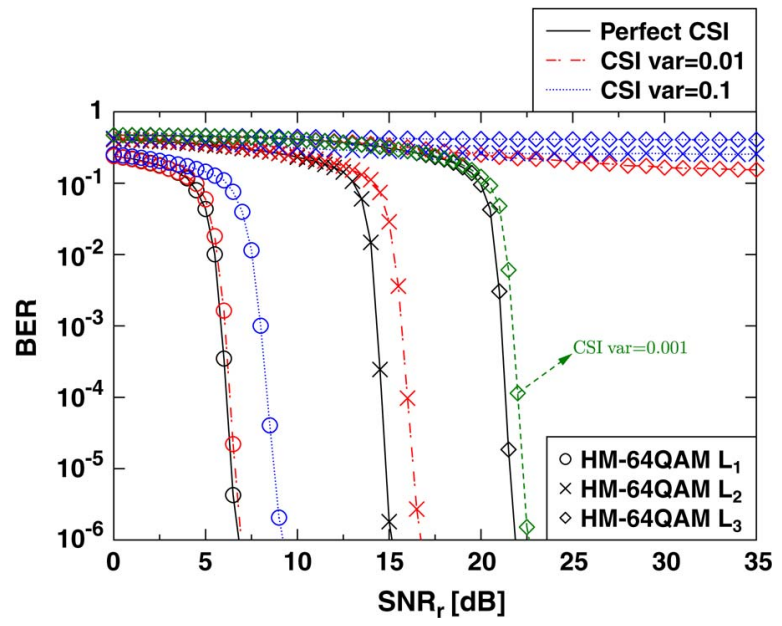

Fig. 8. BER versus SNR performance of the triple-layer coded HM-64QAM scheme with imperfect CSI, where the HM-64QAM ratio pair is $\left(R_{1}=\right.$ $\left.1.5, R_{2}=0.8\right)$. Explicitly, the number of iterations of the rate-1/2 TTCM decoder is $\zeta=4$, the block size is $\eta=12,000$ symbols and an uncorrelated Rayleigh fading channel is considered.

tunnels between the two 'Inner' curves and the $\zeta=4$-iteration 'Outer' curve. Moreover, both 'Inner' curves are very close to the $\zeta=4$-iteration 'Outer' curve, since the DN fully detects the SPM-16QAM symbols for the sake of receiving the softinformation of $L_{2}$ and $L_{3}$. We have investigated the optimum number of iterations for the TTCM decoder. It can be observed from Fig. 7 that increasing $\zeta$ beyond 4 only gives us a marginal gain, while significantly increasing the decoding complexity. Hence we have opted for $\zeta=4$ for our design.

\section{B. Imperfect CSI for Receiving Triple-Layer HM and Pre-Coding Based Twin-Layer SPM}

When considering the impact of imperfect CSI at all nodes in cooperative communications, the performance of our coherent scheme is expected to be degraded. To investigate the robustness of our triple-layer coded HM-64QAM and pre-coding based twin-layer coded SPM-16QAM schemes, we model the CSI estimation errors by a Gaussian process superimposed on each channel coefficient at the two RNs and DN, where the CSI estimation error variance is denoted by $\widetilde{\sigma}$.

The BER performance of receiving triple-layer coded HM-64QAM recorded for diverse CSI estimation error variances $\widetilde{\sigma}$ is shown in Fig. 8. We can observe that a CSI estimation error of $\widetilde{\sigma}=0.01$ only slightly impedes the performance of receiving $L_{1}$, but will cause a $2 \mathrm{~dB}$ SNR degradation (at a BER of $10^{-6}$ ) for receiving $L_{2}$. However, the system excessively degrade $L_{3}$ for $\widetilde{\sigma}=0.01$. By contrast, a CSI estimation error of $\widetilde{\sigma}=0.1$ would impose a $5 \mathrm{~dB}$ SNR degradation on the performance of $L_{1}$, whilst the cooperative communication system will have completely lost $L_{2}$ and $L_{3}$ in this situation. It is shown in Fig. 8 that a CSI estimation error variance below $\widetilde{\sigma}=0.001$ is required for receiving $L_{3}$. Hence, we find that the robustness of each layer in the HM-64QAM symbols against imperfect CSI is different, where $L_{1}$ is the most robust layer, while $L_{3}$ has the highest sensitivity to CSI errors.

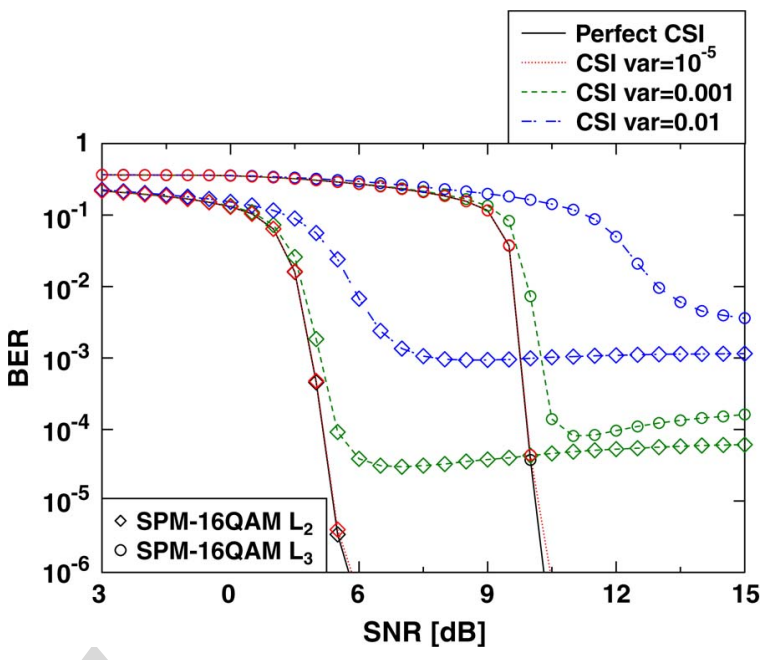

Fig. 9. BER versus SNR performance of the twin-layer coded SPM-16QAM scheme with imperfect CSI, where the SPM-16QAM weighting pair is $(\alpha=$ 0.87, $\beta=0.5$ ). Explicitly, the number of iterations of the rate-1/2 TTCM decoder is $\zeta=4$, the block size is $\eta=12,000$ symbols and an uncorrelated Rayleigh fading channel is considered.

The performance of our pre-coding based coded SPM scheme associated with CSI estimation errors are shown in Fig. 9. If there is CSI estimation error for the $R_{1} N\left(R_{2} N\right)$ and the DN link, the resultant $\rho_{1}$ in (7) and $\rho_{2}$ in (8) become:

$$
\begin{gathered}
\rho_{1}=\frac{\widetilde{h}_{R_{1} D}^{*}}{\left|\widetilde{h}_{R_{1} D}^{*}\right|^{2} \sqrt{G_{R_{1} D}}}, \\
\rho_{2}=\frac{\widetilde{h}_{R_{2} D}^{*}}{\left|\widetilde{h}_{R_{2} D}^{*}\right|^{2} \sqrt{G_{R_{2} D}}} .
\end{gathered}
$$

Hence, the pre-coding based twin-layer coded SPM-16QAM symbols received by the DN during the second TS is expressed as:

$$
y_{R D}=\frac{\alpha \widetilde{h}_{R_{1} D}^{*} h_{R_{1} D}}{\left|\widetilde{h}_{R_{1} D}^{*}\right|^{2}} x_{2}+\frac{\beta \widetilde{h}_{R_{2} D}^{*} h_{R_{2} D}}{\left|\widetilde{h}_{R_{2} D}^{*}\right|^{2}} x_{3}+n_{R D} .
$$

More specifically, we have:

$$
\begin{aligned}
& \widetilde{h}_{R_{1} D}=h_{R_{1} D}+\Delta_{1}, \\
& \widetilde{h}_{R_{2} D}=h_{R_{2} D}+\Delta_{2},
\end{aligned}
$$

where $\Delta_{1}$ is the CSI estimation error imposed on $h_{R_{1} D}$, and $\Delta_{2}$ is the CSI error contaminating $h_{R_{2} D}$. In order to simplify our discussions, we assume that both $\Delta_{1}$ and $\Delta_{2}$ obey the Gaussian distribution with zero mean and a variance of $\widetilde{\sigma}_{1}=\widetilde{\sigma}_{2}=\widetilde{\sigma}$. It can be seen from Fig. 9 that our pre-coding based twin-layer coded SPM-16QAM scheme is sensitive to the CSI estimation errors. Explicitly, an error floor around BER of $10^{-4}$ exists for the detection of $L_{2}$ and $L_{3}$ of the twin-layer SPM scheme when $\widetilde{\sigma}=0.001$. Upon increasing the CSI estimation error variance $\widetilde{\sigma}$, the performance of the pre-coding based coded SPM scheme will be dramatically reduced. The error floor will be eliminated at BER of $10^{-6}$ by employing sophisticated channel estimation 


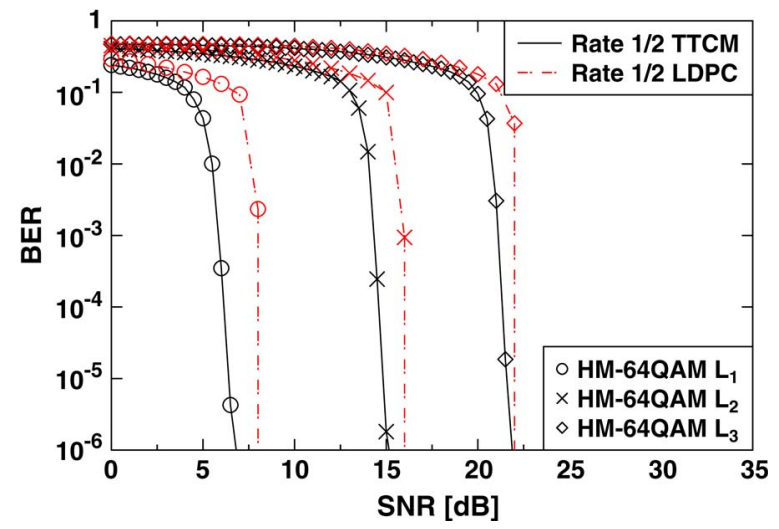

Fig. 10. The BER versus SNR performance of our triple-layer 64QAM TTCHM scheme and triple-layer 64QAM LDPC scheme. The triple-layer HM-64QAM ratio pair is $\left(R_{1}=1.5, R_{2}=0.8\right)$. The block size of the decoder of the two coding schemes is the same, which is $\eta=12,000$ symbols, the code-rate of the two coding schemes' encoder is $1 / 2$. For TTCM decoder, the iteration number is $\zeta=4$ and the maximum iteration number of LDPC decoder is $\zeta_{l}=20$. The system communicates over uncorrelated Rayleigh fading channel.

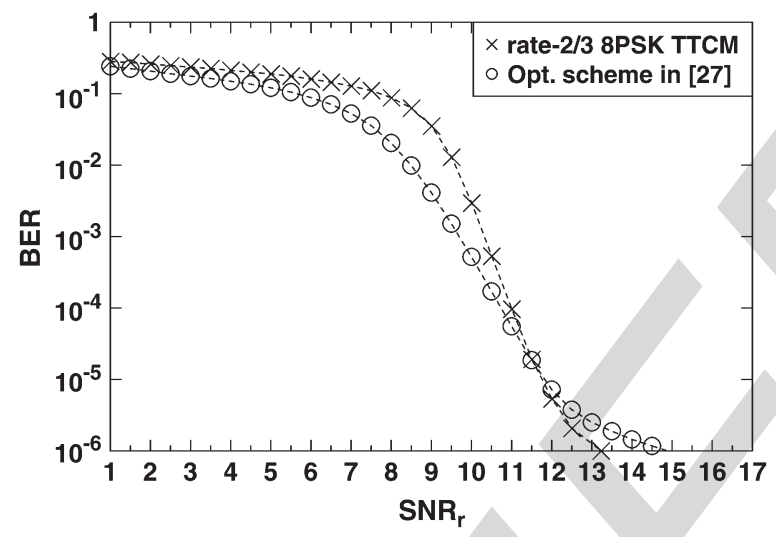

Fig. 11. The BER versus SNR performance of non-cooperative rate-2/3 8PSK TTCM and the optimized scheme in [26]. The number of iterations of the TTCM decoder in each of the three schemes is $\zeta=4$, the block size is $\eta=12,000$ symbols. An uncorrelated Rayleigh fading channel is considered.

schemes, which could reduce $\widetilde{\sigma}$ below a level of $10^{-5}$, as shown in Fig. 9.

\section{Comparisons With Other Systems}

Fig. 10 compares our rate-1/2 TTCM aided triple-layer HM-64QAM scheme to that of the same system but aided by a rate-1/2 LDPC code [38]. It can be observed that the bitbased LDPC coding scheme performs slightly worse than the symbol based TTCM coding scheme, even though the number of LDPC decoder iterations is much higher than that of the TTCM decoder. This is because a symbol-based scheme tends to have a lower convergence SNR than an equivalent bit-based scheme, as detailed in [39].

The BER performance curves of the non-cooperative rate- $2 / 3$ 8PSK TTCM and of our scheme in [26] are shown in Fig. 11. The throughputs of the two schemes are $2 \mathrm{bps}$ and $1.5 \mathrm{bps}$ (the scheme in [26] requires 2 TSs for cooperative transmission). Note that the throughput of the optimized scheme proposed in this paper is 1.455 bps. By contrast, the achievable throughput of the system proposed in this paper is $3 \times 0.97=2.91 \mathrm{bps}$, when the channel SNR is sufficiently high for the DN to detect all the three layers of the HM symbols in a single TS without the assistance of the RN. The $\mathrm{SNR}_{t}^{S N}$ required for achieving a BER of $10^{-6}$ for the non-cooperative rate-2/3 8PSK is $13.2 \mathrm{~dB}$, while that of the optimized scheme in [26] is $14.8 \mathrm{~dB}$. By contrast, the $\mathrm{SNR}_{t}^{S N}$ of the optimized scheme proposed in this paper is $6.81 \mathrm{~dB}$ and $\overline{S N R_{t}}$ is $6.94 \mathrm{~dB}$ per TS. Additionally, the simulation results of [13] show that in order to transmit a twin-layer HM-16QAM signal with the aid of two rate-1/2 H264/AVC encoders over an AWGN channel for achieving a BER of $10^{-6}$, their $\mathrm{SNR}_{t}^{S N}$ should be higher than $14 \mathrm{~dB}$, which is about $7.19 \mathrm{~dB}$ higher than that of our optimized scheme transmitting a triple-layer HM-64QAM signal over uncorrelated Rayleigh channels. It can be observed that the scheme optimized in this paper reduced the $\mathrm{SNR}_{t}^{S N}$ and $\overline{S N R_{t}}$ of the cooperative system.

\section{CONCLUSION}

In this paper, we proposed a TTCHM aided cooperative communication system. The system employed both pre-coding and SPM schemes at the two RNs, as detailed in Section II. The results have demonstrated that the best performance of the system is achieved at a HM-64QAM ratio pair of $\left(R_{1}=1.5\right.$, $\left.R_{2}=0.8\right)$ and the optimum SPM ratio pair is $(\alpha=0.87, \beta=$ 0.5 ). The optimized system requires an $\overline{S N R_{t}}$ of $6.94 \mathrm{~dB}$ per TS. It can be concluded that by employing HM in cooperative communications, both the $\operatorname{SNR}_{t}^{S N}$ may indeed be reduced, along with the $\overline{S N R_{t}}$ of the entire system. We note that spatial modulation [40] may also be employed for further reducing the transmit power dissipation of the entire system. The benefit of employing spatial modulation will be investigated in our future work.

\section{A. Derivation of (14)}

\section{APPENDIX}

Before normalization, the $L_{1}$ is represented as conventional square 4QAM symbols, hence we may have $d_{0}+d_{1}=\sqrt{2}$. Therefore, it can be expressed that:

$$
\delta_{1}=\frac{d_{0}}{2}=\frac{d_{0}}{\sqrt{2} \sqrt{2}}=\frac{d_{0}}{\sqrt{2}\left(d_{0}+d_{1}\right)}=\frac{1}{\sqrt{2}\left(1+R_{1}\right)} .
$$

\section{B. Derivation (15)}

As shown in Fig. 3, we have:

$$
R_{1}=\frac{d_{1}}{d_{0}} \quad \text { and } \quad R_{2}=\frac{d_{3}}{d_{2}} .
$$

Hence, we can write $d_{1}, d_{2}$ and $d_{3}$ as:

$$
\begin{aligned}
& d_{1}=d_{0} R_{1} \quad \text { and } \quad d_{3}=d_{2} R_{2}, \\
& d_{1}=d_{3}+2 \delta_{2} \quad \text { and } \quad d_{2}=d_{0}+2 \delta_{2},
\end{aligned}
$$


It may be observed from (56) and (57) that

$$
\delta_{2}=\frac{d_{1}-d_{3}}{2}=\frac{d_{0} R_{1}-d_{2} R_{2}}{2}=\frac{d_{0} R_{1}-\left(d_{0}+2 \delta_{2}\right) R_{2}}{2},
$$

where $\delta_{2}$ here may be expressed as:

$$
\delta_{2}=\frac{d_{0}\left(R_{1}-R_{2}\right)}{2\left(1+R_{2}\right)} .
$$

Upon substituting (54) into (59) we have:

$$
\delta_{2}=\frac{\left(R_{1}-R_{2}\right)}{\sqrt{2}\left(1+R_{1}\right)\left(1+R_{2}\right)} .
$$

\section{Restrictions on $R_{1}$}

It can be observe that:

$$
\begin{aligned}
& R_{1: \max }=\frac{d_{1_{\text {max }}}}{d_{0_{\text {min }}}}=\frac{\sqrt{2}}{0} \Rightarrow \infty, \\
& R_{1: \min }=\frac{d_{1_{\text {min }}}}{d_{0_{\max }}}=\frac{0}{\sqrt{2}} \Rightarrow 0,
\end{aligned}
$$

so we have $0<R_{1}<\infty$.

\section{Restrictions on $R_{2}$}

Note that $R_{2}$ is directly restricted by $R_{1}$ as follows:

$$
R_{2_{\max }}=\frac{d_{3_{\max }}}{d_{2_{\min }}}=\frac{d_{1}}{d_{0}} \Rightarrow R_{1} .
$$

If $R_{1}>1$, then $\max \left(\delta_{2}\right) \rightarrow d_{0} / 2$ and we have:

$$
R_{2_{\min }}=\frac{d_{3_{\min }}}{d_{2_{\max }}}=\frac{d_{1}-d_{0}}{2 d_{0}} \Rightarrow \frac{1}{2}\left(R_{1}-1\right) .
$$

By contrast, if $R_{1}<1$, then we have $\max \left(\delta_{2}\right) \rightarrow d_{3} / 2$ and hence:

$$
R_{2_{\min }}=\frac{d_{3_{\min }}}{d_{2_{\max }}}=\frac{0}{d_{0}+d_{3}} \Rightarrow 0 .
$$

\section{REFERENCES}

[1] C. Hellge, S. Mirta, T. Schierl, and T. Wiegand, "Mobile TV with SVC and hierarchical modulation for DVB-H broadcast services," in Proc. IEEE Int. Symp. BMSB, May 2009, pp. 1-5.

[2] S. Wang, S. Kwon, and B. K. Yi, "On enhancing hierarchical modulation," in Proc. IEEE Int. Symp. Broadband Multimedia Syst. Broadcast., Jun. 2008, pp. 1-6.

[3] R. Y. Kim and Y.-Y. Kim, "Symbol-level random network coded cooperation with hierarchical modulation in relay communication," IEEE Trans. Consum. Electron., vol. 55, no. 3, pp. 1280-1285, Oct. 2009.

[4] H. Jiang and P. A. Wilford, "A hierarchical modulation for upgrading digital broadcast systems," IEEE Trans. Broadcast., vol. 51, no. 2, pp. $223-$ 229, Jun. 2005.

[5] M. K. Chang and S. Y. Lee, "Performance analysis of cooperative communication system with hierarchical modulation over Rayleigh fading channel," IEEE Trans. Wireless Commun., vol. 8, no. 6, pp. 2848-2852, Jun. 2009.

[6] S. Y. Lee and K. C. Whang, "A collaborative cooperation scheme using hierarchical modulation," in Proc. IEEE 68th VTC, Sep. 2008, pp. 1-5.
[7] Z. Xuehua, A. Ghrayeb, and M. Hasna, "On hierarchical network coding versus opportunistic user selection for two-way relay channels with asymmetric data rates," IEEE Trans. Commun., vol. 61, no. 7, pp. 2900-2910, Jul. 2013.

[8] H. Mukhtar and M. EI-Tarhuni, "An adaptive hierarchical QAM scheme for enhanced bandwidth and power utilization," IEEE Trans. Commun., vol. 60, no. 8, pp. 2275-2284, Aug. 2012.

[9] K. Ramchandran, A. Ortega, K. M. Uz, and M. Vetterli, "Multiresolution broadcast for digital HDTV using joint source/channel coding," IEEE $J$. Sel. Areas Commun., vol. 11, no. 1, pp. 6-23, Jan. 1993.

[10] S. H. Chang, R. Minjoong, P. C. Cosman, and L. B. Mistein, "Optimized unequal error protection using multiplexed hierarchical modulation," IEEE Trans. Inf. Theory, vol. 58, no. 9, pp. 5816-5840, Sep. 2012.

[11] Y. J. Noli, H. C. Lee, and L. Y. Lee, "Design of unequal error protection for MIMO-OFDM systems with hierarchical signal constellations," J. Commun. Netw., vol. 9, no. 2, pp. 167-176, Jun. 2007.

[12] Y. C. Chang, S. W. Lee, and R. Komiya, "A low complexity hierarchical QAM symbol bits allocation algorithm for unequal error protection of wireless video transmission," IEEE Trans. Consum. Electron., vol. 55, no. 3, pp. 1089-1097, Aug. 2009.

[13] K. M. Alajel, W. Xiang, and Y. F. Wang, "Unequal error protection scheme based hierarchical 16-QAM for 3-D video transmission," IEEE Trans. Consum. Electron., vol. 58, no. 3, pp. 731-738, Aug. 2012.

[14] S. S. Arslan, P. C. Cosman, and L. B. Milstein, "Coded hierarchical modulation for wireless progressive image transmission," IEEE Trans. Veh. Technol., vol. 60, no. 9, pp. 4299-4313, Nov. 2011.

[15] S. S. Arslan, P. C. Cosman, and L. B. Milstein, "On hard decision upper bounds for coded M-ary hierarchical modulation," in Proc. 45th Аnnu. CISS, Mar. 2011, pp. 1-6.

[16] C. Hausl and J. Hagenauer, "Relay communication with hierarchical modulation," IEEE Commun. Lett., vol. 11, no. 1, pp. 64-66, Jan. 2007.

[17] L. Hanzo, T. H. Liew, B. L. Yeap, R. Y. S. Tee, and S. X. Ng, Turbo Coding, Turbo Equalisation and Space-Time Coding: EXIT-Chart-Aided Near-Capacity Designs for Wireless Channels, 2nd ed. Hoboken, NJ, USA: Wiley-IEEE Press, 2011

[18] C. H. Choi, Y. J. Kim, and G. H. Im, "Bit-interleaved coded transmission with multilevel modulation for non-orthogonal cooperative systems," IEEE Trans. Commun., vol. 59, no. 1, pp. 95-105, Jan. 2011.

[19] Z. Y. Li, M. Peng, and W. B. Wang, "Hierarchical modulated channel and network coding scheme in the multiple-access relay system," in Proc. IEEE 13th ICCT, Sep. 2011, pp. 984-988.

[20] J. M. Park, S. L. Kim, and J. H. Choi, "Hierarchically modulated network coding for asymmetric two-way relay systems," IEEE Trans. Veh. Technol., vol. 59, no. 5, pp. 2179-2184, Jun. 2010.

[21] Z. G. Du, P. L. Hong, K. P. Xue, and J. L. Peng, "Hierarchically modulated coded cooperation for relay system," in Proc. IEEE CCNC, Jan. 2012, pp. $812-816$.

[22] D. K. Kwon, W. J. Kim, K. H. Suh, and H. Lim, "A higher data-rate T-DMB system based on a hierarchical A-DPSK modulation," IEEE Trans. Broadcast., vol. 55, no. 1, pp. 42-50, Mar. 2009.

[23] P. Robertson and T. Worz, "Bandwidth-efficient turbo trellis-coded modulation using punctured component codes," IEEE J. Sel. Areas Commun., vol. 16, no. 2, pp. 206-218, Feb. 1998.

[24] S. X. Ng, J. Y. Chung, and L. Hanzo, "Turbo-detected unequal protection MPEG-4 wireless video telephony using multi-level coding, trellis coded modulation and space-time trellis coding," IEE Proc. Commun., vol. 152, no. 6, pp. 1116-1124, Dec. 2005.

[25] S. X. Ng, C. Y. Qian, D. D. Liang, and L. Hanzo, "Adaptive turbo trellis coded modulation aided distributed space-time trellis coding for cooperative communications," in Proc. IEEE 71st VTC-Spring, May 2010, pp. 1-5.

[26] H. Sun, Y. R. Shen, S. X. Ng, and L. Hanzo, "Turbo trellis coded hierarchical modulation for cooperative communications," in Proc. WCNC, Apr. 2013, pp. 2789-2794.

[27] S. H. Chang, R. Minijoong, P. C. Cosman, and L. B. Milstein, "Superposition MIMO coding for the broadcast of layered sources," IEEE Trans. Commun., vol. 59, no. 12, pp. 3240-3248, Dec. 2011.

[28] Y. K. Huo, M. El-Hajjar, and L. Hanzo, "Inter-layer FEC aided unequal error protection for multilayer video transmission in mobile TV," IEEE Trans. Circuits Syst. Video Technol., vol. 23, no. 9, pp. 1622-1634, Sep. 2013.

[29] H. Ochiai, P. Mitran, and V. Tarokh, "Design and analysis of collaborative diversity protocols for wireless sensor networks," in Proc. IEEE 60th VTC-Fall, Sep. 2004, vol. 7, pp. 4645-4649.

[30] C. Xu, S. X. Ng, and L. Hanzo, "Near-capacity irregular convolutional coded cooperative differential linear dispersion codes using 
multiplesymbol differential detection," IEEE Signal Process. Lett., vol. 18, no. 3, pp. 4645-4649, Mar. 2011.

[31] S. X. .Ng, Y. H. Li, and L. Hanzo, "Distributed turbo trellis coded modulation for cooperative communications," in Proc. ICC, Jun. 2009, pp. 1-5.

[32] A. Goldsmith, Wireless Communications, 1st ed. Cambridge, U.K.: Cambridge Univ. Press, 2005

[33] S. M. Ross, Introduction to Probability Models, 9th ed. New York, NY, USA: Academic, 2007.

[34] H. Sun, S. X. Ng, and L. Hanzo, "Superposition coded modulation for cooperative communications," in Proc. IEEE VTC Fall, Sep. 2012, pp. $1-5$.

[35] A. Papoulis, Probability, Random Variables and Stochastic Processes, 4th ed. New York, NY, USA: McGraw-Hill, Jan. 2002.

[36] S. ten Brink, "Convergence behavior of iteratively decoded parallel concatenated codes," IEEE Trans. Commun., vol. 49, no. 10, pp. 1727-1737, Aug. 2002.

[37] S. X. Ng, J. Wang, and L. Hanzo, "Unveiling near-capacity code design: The realization of shannon's communication theory for MIMO channels," in Proc. IEEE ICC, May 2008, pp. 1415-1419.

[38] R. G. Gallager, "Low-density parity-check codes," IRE Trans. Inf. Theory, vol. 8, no. 1, pp. 21-28, Jan. 1962.

[39] B. Scanavino, G. Montorsi, and S. Benedetto, "Convergence properties of iterative decoders working at bit and symbol level," in Proc. IEEE GLOBECOM, Nov. 2001, vol. 2, pp. 1037-1041.

[40] M. D. Renzo, H. Haas, A. Ghrayeb, S. Sugiura, and L. Hanzo, "Spatial modulation for generalized MIMO: challenges, opportunities, implementation," Proc. IEEE, vol. 102, no. 1, pp. 56-103, Jan. 2014.

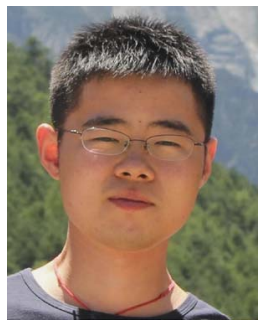

Hua Sun received the B.Eng. degree in electronics and information engineering from Huazhong University of Science and Technology, Wuhan, China, in 2009 and the M.Sc. degree (with distinction) in wireless communications from the University of Southampton, Southampton, U.K., in 2010. $\mathrm{He}$ is currently working toward the Ph.D. degree from the Research Group of Communications, Signal Processing and Control, School of Electronics and Computer Science, University of Southampton, Southampton, U.K. His research interests include superposition modulation, hierarchical modulation, turbo trellis-coded modulation, and cooperative communications.

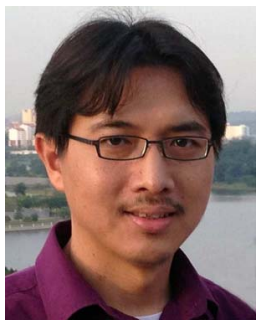

Soon Xin Ng (S'99-M'03-SM'08) received the B.Eng. degree (first class honor) in electronics engineering and the Ph.D. degree in wireless communications from the University of Southampton, Southampton, U.K., in 1999 and 2002, respectively. From 2003 to 2006, he was a Postdoctoral Research Fellow working on collaborative European research projects known as SCOUT, NEWCOM, and PHOENIX. Since August 2006, he has been with the School of Electronics and Computer Science, University of Southampton, where he is currently an Associate Professor. He is involved in the OPTIMIX and CONCERTO European projects, and the IU-ATC and UC4G projects. He is the author of over 180 papers and is the coauthor of two John Wiley/IEEE Press books in this field. His research interests include adaptive coded modulation, coded modulation, channel coding, space-time coding, joint source and channel coding, iterative detection, OFDM, MIMO, cooperative communications, distributed coding, quantum error correction codes and joint wireless-and-optical-fiber communications. He is a Chartered Engineer and a Fellow of the Higher Education Academy in the U.K.

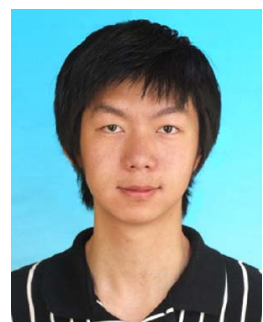

Chen Dong received the B.S. degree in electronic information sciences and technology from the University of Science and Technology of China, Hefei, China, in 2004; the M.Eng. degree in pattern recognition and automatic equipment from the University of Chinese Academy of Sciences, Beijing, China, in 2007; and the Ph.D. degree from the University of Southampton, Southampton, U.K. He is currently a Postdoctoral Researcher with the University of Southampton. His research interests include applied math, relay systems, channel modeling, and crosslayer optimization. He received a scholarship under the U.K.-China Scholarships for Excellence Programme and the Best Paper Award at the 2014 Fall IEEE Vehicular Technology Conference.

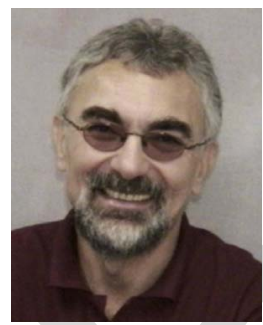

Lajos Hanzo (F'04) received the Master's degree in electronics, the Ph.D. degree, and the Doctor Honoris Causa degree from the Technical University of Budapest, Budapest, Hungary, in 1976, 1983, and 2009, respectively.

During his 38-year career in telecommunications, he has held various research and academic posts in Hungary, Germany, and U.K. Since 1986, he has been with the School of Electronics and Computer Science, University of Southampton, Southampton, U.K., where he is currently the Chair in telecommunications. He is also a Chaired Professor with Tsinghua University, Beijing, China. He has successfully supervised about $100 \mathrm{Ph}$.D. students, coauthored 20 John Wiley/IEEE Press books on mobile radio communications totalling in excess of 10000 pages, and published more than 1400 research entries at IEEE Xplore. Currently, he is directing a 100-strong academic research team, working on a range of research projects in the field of wireless multimedia communications sponsored by industry, the Engineering and Physical Sciences Research Council (EPSRC) U.K., the European Research Council through an Advanced Fellow Grant, and the Royal Society through the Wolfson Research Merit Award. He is an enthusiastic supporter of industrial and academic liaison, and he offers a range of industrial courses

Dr. Hanzo has acted both as Technical Program Committee and General Chair of IEEE conferences, presented keynote lectures, and received a number of distinctions. He is also a Governor of the IEEE Vehicular Technology Society. From 2008 to 2012, he was the Editor-in-Chief of the IEEE Press. His research is funded by the European Research Council's Senior Research Fellow Grant. He is a Fellow of the Royal Academy of Engineering, Institution of Engineering and Technology, and European Association for Signal Processing. 


\title{
Decode-and-Forward Cooperation-Aided Triple-Layer Turbo-Trellis-Coded Hierarchical Modulation
}

\author{
Hua Sun, Soon Xin Ng, Senior Member, IEEE, Chen Dong, and Lajos Hanzo, Fellow, IEEE
}

\begin{abstract}
Hierarchical modulation (HM) is widely employed across the telecommunication industry. The potential application of the coded HM scheme in cooperative communications has drawn much interest. In this paper, a twin-relay-aided triple-layer cooperative communication system is proposed. The system amalgamates rate-1/2 TTCM, triple-layer HM-64QAM, and twin-layer SPM-16QAM schemes in the context of cooperative communications. We have optimized the entire system based on the HM ratio pair $\left(R_{1}, R_{2}\right)$, the superposition modulation (SPM) weighting pair $(\alpha, \beta)$, and the positions of the two relays. The simulation results show that our optimized system is capable of reliably transmitting a triple-layer HM-64QAM signal with the aid of two time slots at an average signal-to-noise ratio of $6.94 \mathrm{~dB}$ per time slot.
\end{abstract}

Index Terms-Hierarchical modulation, superposition modulation, turbo trellis-coded modulation, cooperative communication, soft decoding and power efficiency.

\section{INTRODUCTION}

$\mathbf{H}$ IERARCHICAL modulation (HM) constitutes an integral part of the DVB-T/-H standard [1], but it has also been widely employed by the IT industry for upgrading diverse telecommunication services [2], [3]. Compared to a system using conventional modulation, the system employing HM has a higher flexibility, while maintaining backward compatibility. Explicitly, both the original and the upgraded new services may be combined by the HM scheme and broadcast to the receivers without requiring any additional bandwidth. Although the services is upgraded to a higher data rate, the original devices are still supported by the upgraded broadcast system without requiring software or hardware upgrade [4].

HM has been developed for combining independent information streams at bit-level layer by layer, which are then mapped onto HM constellations. The information contained in different

Manuscript received July 9, 2014; revised November 5, 2014; accepted December 19, 2014. This work was supported in part by the European Research Council under the Advanced Fellowship Grant, by the EPSRC through IndiaU.K. Advanced Technology Centre, and by the EU's Concerto Project. The associate editor coordinating the review of this paper and approving it for publication was G. Bauch.

The authors are with the School of Electronics and Computer Science, University of Southampton, Southampton SO17 1BJ, U.K.

Color versions of one or more of the figures in this paper are available online at http://ieeexplore.ieee.org.

Digital Object Identifier 10.1109/TCOMM.2014.2387164 layers may also be demapped/detected separately. The general performance of the HM scheme have been detailed in [5]-[8]. It can be observed from the simulation results of [5], [6] that different layers in HM constellations receive different protection levels. Consequently, the required received Signal-to-Noise Ratio $\left(\mathrm{SNR}_{r}\right)$ for receiving the layer with higher protection level requires lower $\mathrm{SNR}_{r}$ than that of the less protected layers. The author of [9] employed HM in his system to provide unequal error protection (UEP) for the information contained in different layers. It has drawn a lot of interests [10]-[15]. More specifically, the authors of [13], [14] invoked a HM scheme for providing UEP for video and image encoding, where the information bits are mapped to specific protection layers according to their error-sensitivity-based priority. Moreover, the HM scheme has also been combined with sophisticated channel coding schemes in [13], [14], for protecting the most important information. The simulation results of [13], [14] have shown that receiving the information having the highest priority requires a lower $\mathrm{SNR}_{r}$ compared to conventional modulation schemes at a given target BER performance.

A typical relay aided coded HM scheme was introduced in [16], where Hausl and Hagenauer combined Turbo Coding (TC) [17] with a HM scheme conceived for cooperative communications, where the original signal sequence was broadcast by the Source Node (SN) by ensuring that the layer with higher protection may be received by the Destination Node (DN) directly, while the less protected layer will be received and retransmitted by the Relay Node (RN). However, the authors of [16] only considered the specific scenario, when the position of the RN is right in the middle of the SN-DN path and invoked a specific bit-to-symbol mapping scheme. The performance of the coded HM schemes was then further discussed in [18][21] in the context of cooperative communications, where the common choice is to employ multiple encoders at the $\mathrm{SN}$ and combine all the coded bit sequences layer-by-layer to create a HM signal sequence. Again, the less well-protected layers are assisted by the RN of the cooperative network.

The bit-to-symbol mapping optimization of the HM scheme was considered in [20], [22]. More specifically, by appropriately designing the constellation mapping, the HM scheme is capable of enhancing the protection of the higher-priority information at the expense of providing a weaker protection for the other layers. In [21], the specific position of the $\mathrm{RN}$ was explicitly considered in the BER analysis. For a specific coded $\mathrm{HM}$ scheme aided system, the receive power at the RN should 
be sufficiently high for guaranteeing that the RN becomes capable of receiving the information in the lower protected layer with an acceptable integrity. Therefore, the position of the RN may influence the power allocation of the entire system. Several parameters have to be taken into consideration, when optimizing a coded HM aided cooperative communication system. On one hand, distorting the HM constellation for the sake of improving the BER of its high-priority layers at the detriment of its low-priority layers degrades its average BER, compared to conventional modulation schemes. On the other hand, sophisticated channel coding schemes, such as TrellisCoded Modulation (TCM), Turbo Trellis-Coded Modulation (TTCM) and Bit-Interleaved Coded Modulation (BICM) [17], [23]-[25] are required for protecting each HM layer at the expense of an increased complexity. Hence, giving cognizance both to the complexity and to the power efficiency of the overall system, while maintaining its flexibility becomes a challenging task.

In [26], a cooperative communication system assisted by a TTCM based HM-16QAM scheme was proposed, where we proposed the optimum constellation mapping for the HM-16QAM scheme in the context of single RN aided cooperative communications. However, the position of the RN was fixed to be right in the middle of the SN-DN path. Hence, the scheme of [26] was suboptimal, because its power allocation was suboptimal. Against this background, in this treatise we intrinsically amalgamate HM, Superposition Modulation (SPM) [27] and TTCM for creating an attractive cooperative communication system. Our goal is to increase the time-efficiency and reduce the total power consumption of the entire system, while maintaining a low complexity. This cooperative communication system model may be readily used for assisting multilayer video transmission for example [13], [28] or for multilayer image transmission [14]. We exploit the idealized simplifying assumption that the system benefits from perfect Channel State Information (CSI), including both the fading and path-loss. Hence, according to the receiver's SNR, we are capable of determining the transmission power required at the transmitter, which we defined as the transmit SNR $\left(\mathrm{SNR}_{t}\right)^{1}$. We proposed a Turbo Trellis-Coded Hierarchical Modulation (TTCHM) aided twin-relay based cooperative communication scheme, where three rate-1/2 TTCM encoders are employed at the $\mathrm{SN}$ for constructing a 64QAM-based triple-layer HM scheme. The TTCM scheme detailed in [17] has a better performance for transmission over Rayleigh fading channels than other joint coding and modulation schemes, such as TCM and BICM. An excellent performance can be attained by TTCM without expanding the bandwidth. A rate-k/(k+1) TTCM scheme can be used for protecting a k-bit HM layer by expanding the number of constellation points from $2^{k}$ to $2^{k+1}$. We considered $k=1$ in this contribution.

Depending on the specific symbol-to-bit demapping arrangement of the HM scheme, different HM layers have different

\footnotetext{
${ }^{1}$ The definition of transmit SNR was proposed in [29], which is convenient for simplifying the discussions, although this is not a physically measurable quantity, because it relates the power at the transmitter to the noise at the receivers.
}

protections. Explicitly, the information in the higher-protection layers may require a lower $\mathrm{SNR}_{r}$ at the $\mathrm{DN}$ than that of the information in the lower-protection layers. Hence, when the SN transmits a multi-layer $\mathrm{HM}$ signal in our cooperative communication network, the $\mathrm{SNR}_{t}$ at the $\mathrm{SN}\left(\mathrm{SNR}_{t}^{S N}\right)$ may be reduced to the minimum value that can 'just' guarantee the successful detection of the base layer (highest priority) of the $\mathrm{HM}$ signal at the DN. By contrast, the information in the lower priority layers may be received and retransmitted by the RN. Since we proposed a triple-layer HM scheme, two RNs are activated for retransmitting the information of the two lower layers. More specifically, a linear SPM scheme is employed by the two RNs for simultaneously transmitting the two signal frames to the DN during the second time slot (TS). Hence, two TSs are required for the transmission of all the three layers from the SN to the DN. Since the transmissions between the SN and $\mathrm{RN}$ (or RN and DN) only deal with a single 4QAM layer of the triple-layer HM-64QAM signals, the decoding complexity imposed on the two RNs (and DN) is reduced. Moreover, the $\mathrm{SNR}_{t}$ at the $\mathrm{RN}\left(\mathrm{SNR}_{t}^{R N}\right)$ can also be minimized, because both RNs will only retransmit using 4QAM. If only one RN is available for assisting the transmissions, the $\mathrm{RN}$ would have to detect both enhancement layers from the HM-64QAM signals. The position of this RN would be near to the $\mathrm{SN}$ and the transmission between the RN and the $\mathrm{DN}$ will be based on 16QAM modulation. This would require a high $\mathrm{SNR}_{t}$ due to the transmission of a higher-order modulation scheme over a longer distance. Due to the flexibility of HM, the lowestprotection layer that contains the least important information can be discarded in the adverse situation, when none of the RNs is capable of detecting it. Nonetheless, the DN can still receive the pair of more important layers of the HM-64QAM. We found that apart from reducing the power dissipation of the entire system, the processing complexity of the twin-relay aided cooperative communication network may also be mitigated, when an appropriate design is invoked.

The main contributions of this paper are as follows:

- A triple-layer HM-64QAM scheme is designed for aiding a Decode-and-Forward (DF) based cooperative communications, which involves intrinsically amalgamating TTCM, HM and SPM schemes;

- Based on our Monte-Carlo simulations, a power-allocation is conceived and it is demonstrated that the power consumption of the entire system may be readily optimized by relying on the related variables, namely by the HM-based symbol-energy ratio pair $\left(R_{1}, R_{2}\right)$, by the SPM weighting pair $(\alpha, \beta)$, and by the relay's geographic position.

The rest of the paper is organized as follows. Section II introduces both the system model and our cooperative communication strategy. The specific HM-64QAM mapping rule designed for cooperative communication is detailed in Section III. The protocol of the symbol-to-bit demapper of the HM symbols is discussed in Section IV. The triple-layer TTCHM-64QAM system design is detailed in Section V and the simulation results are displayed in Section VI, our conclusions and future research ideas are discussed in Section VII. 


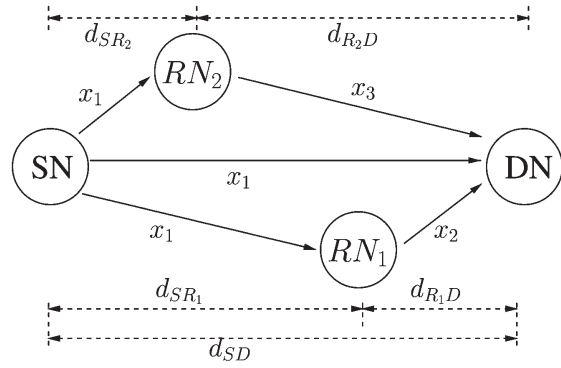

Fig. 1. The model of a two-relay cooperative system.

\section{System Model}

Our TTCHM aided DF RN based cooperative communication system is illustrated in Fig. 1. During the first transmission TS, the $\mathrm{SN}$ will broadcast a sequence of TTCHM symbols $\left\{x_{1}\right\}$ to $\mathrm{RN}_{1}, \mathrm{RN}_{2}$ and $\mathrm{DN}$. In the following TS, $\mathrm{RN}_{1}$ will transmit a signal frame $\left\{x_{2}\right\}$ to the $\mathrm{DN}$, another signal frame $\left\{x_{3}\right\}$ will also be sent to the $\mathrm{DN}$ by the $\mathrm{RN}_{2}$ simultaneously. Again, the entire system would require two TSs to convey the triple-layer TTCHM-64QAM symbol based signal frame $\left\{x_{1}\right\}$ to DN.

We considered an uncorrelated Rayleigh flat-fading channel, both the transmitters and receivers were assumed to acquire perfect CSI. During the first TS, each symbol received by the DN may be expressed as:

$$
y_{S D}=\sqrt{G_{S D}} h_{S D} x_{1}+n_{S D},
$$

where each of the symbols received by the $\mathrm{RN}_{1}$ and $\mathrm{RN}_{2}$ are:

$$
\begin{aligned}
& y_{S R_{1}}=\sqrt{G_{S R_{1}}} h_{S R_{1}} x_{1}+n_{S R_{1}}, \\
& y_{S R_{2}}=\sqrt{G_{S R_{2}}} h_{S R_{2}} x_{1}+n_{S R_{2}},
\end{aligned}
$$

where the subscript SD denotes the SN-DN link and the subscript $\mathrm{SR}_{k}$ represents the $\mathrm{SN}-\mathrm{RN}_{k}$ link. By contrast, the symbols received at the DN during the second TS, which are sent by the two RNs, may be expressed as:

$$
y_{R D}=\rho_{1} \alpha \sqrt{G_{R_{1} D}} h_{R_{1} D} x_{2}+\rho_{2} \beta \sqrt{G_{R_{2} D}} h_{R_{2} D} x_{3}+n_{R D},
$$

where $\rho_{1}$ and $\rho_{2}$ are the pre-coding parameters and $(\alpha, \beta)$ is the SPM ratio pair. The subscript $\mathrm{R}_{k} \mathrm{D}$ represents the $\mathrm{RN}_{k}-\mathrm{DN}$ link. Additionally, the notations $h_{S D}, h_{S R_{k}}$ and $h_{R_{k} D}$ denote the complex-valued coefficients of the uncorrelated Rayleigh fading for the different links, $n_{S D}, n_{S R_{k}}$ and $n_{R_{k} D}$ denote the Additive White Gaussian Noise (AWGN) having a variance of $N_{0} / 2$ per dimension. Moreover, the variables $G_{S D}, G_{S R_{k}}$ and $G_{R_{k} D}$ represent the Reduced-Distance-Related-PathlossReduction (RDRPLR) for each link, which we also refer to as the path-gain [25], [29]-[31]. We consider an inverse-power law based non-free-space path-loss model and naturally and we define the path-loss exponent to be 3 which is usually used to simulate the path-loss in urban areas [32]. The path-gain $G_{S D}$ of the SD link is assumed to be unity. Therefore the path-gains of the two SR links are:

$$
G_{S R_{k}}=\left(\frac{d_{S D}}{d_{S R_{k}}}\right)^{3}
$$

and similarly, the path-gains of the two RD links are:

$$
G_{R_{k} D}=\left(\frac{d_{S D}}{d_{R_{k} D}}\right)^{3}
$$

We assume that every node in the cooperative network has perfect CSI. Hence, given a specific path-loss and a RN position, we may both compensate the effect of the path-loss as well as that of the Rayleigh fading with the aid of transmit pre-coding. Specifically, the $\rho_{1}$ and $\rho_{2}$ pre-coding parameters should satisfy:

$$
\begin{aligned}
& \rho_{1}=\frac{h_{R_{1} D}^{*}}{\left|h_{R_{1} D}\right|^{2} \sqrt{G_{R_{1} D}}}, \\
& \rho_{2}=\frac{h_{R_{2} D}^{*}}{\left|h_{R_{2} D}\right|^{2} \sqrt{G_{R_{2} D}}} .
\end{aligned}
$$

Hence, during the second TS, the signal received by the DN may be written as:

$$
y_{R D}=\alpha x_{2}+\beta x_{3}+n_{R D} .
$$

In a realistic situation, there is always a path-loss between the SN and DN, but in order to simplify the system model, we normalized this path-loss to $0 \mathrm{~dB}$. Hence the transmit power at the $\mathrm{SN}\left(\mathrm{SNR}_{t}^{S N}\right)$ would be identical to the power received at the $\mathrm{DN}\left(\mathrm{SNR}_{r}^{D N}\right)$. If the transmissions between the $\mathrm{SN}$ and DN are on a frame-by-frame basis over an uncorrelated Rayleigh fast fading channel, the average received SNR at DN $\left(\overline{S N R_{r}^{D N}}\right)$ would be given by:

$$
\overline{S N R_{r}^{D N}}=E\left(|h|^{2} S N R_{t}\right)=E\left(|h|^{2}\right) S N R_{t}^{S N},
$$

where the $\mathrm{SNR}_{t}^{S N}$ is the transmit SNR defined as the ratio of the transmit power to the noise power at the DN:

$$
S N R_{t}^{S N}=\frac{E\left(|x|^{2}\right)}{N_{0}}=\frac{1}{N_{0}},
$$

where $E\left(|x|^{2}\right)=1$. Furthermore, the uncorrelated Rayleigh fading coefficient $h$ is generated by the complex-valued Gaussian distribution having a zero mean and a variance of one. When the number of uncorrelated Rayleigh fading coefficients we generated is large, we have [33]:

$$
E\left(|h|^{2}\right)=\frac{1}{N} \sum_{k=1}^{N}\left|h_{k}\right|^{2} \approx 1 .
$$

Hence, for a large frame size of $N$ symbols, we may assume that $\mathrm{SNR}_{r}^{D N}$ is equal to the $\mathrm{SNR}_{t}^{S N}$, or equivalently $\overline{S N R_{r}^{D N}}=S N R_{t}^{S N}$.

To be more specific, the information flow of the entire system is illustrated in the block diagram shown in Fig. 2. In our system, the SN employs three rate-1/2 4QAM-TTCM encoders and combines the three independent codeword sequences into a HM signal stream. Thus, the signal frame $\left\{x_{1}\right\}$ is formed by HM-64QAM symbols. When the transmit power at the source is relatively low, the DN may opt for decoding only the information from Encoder 1 during the first TS, where the 


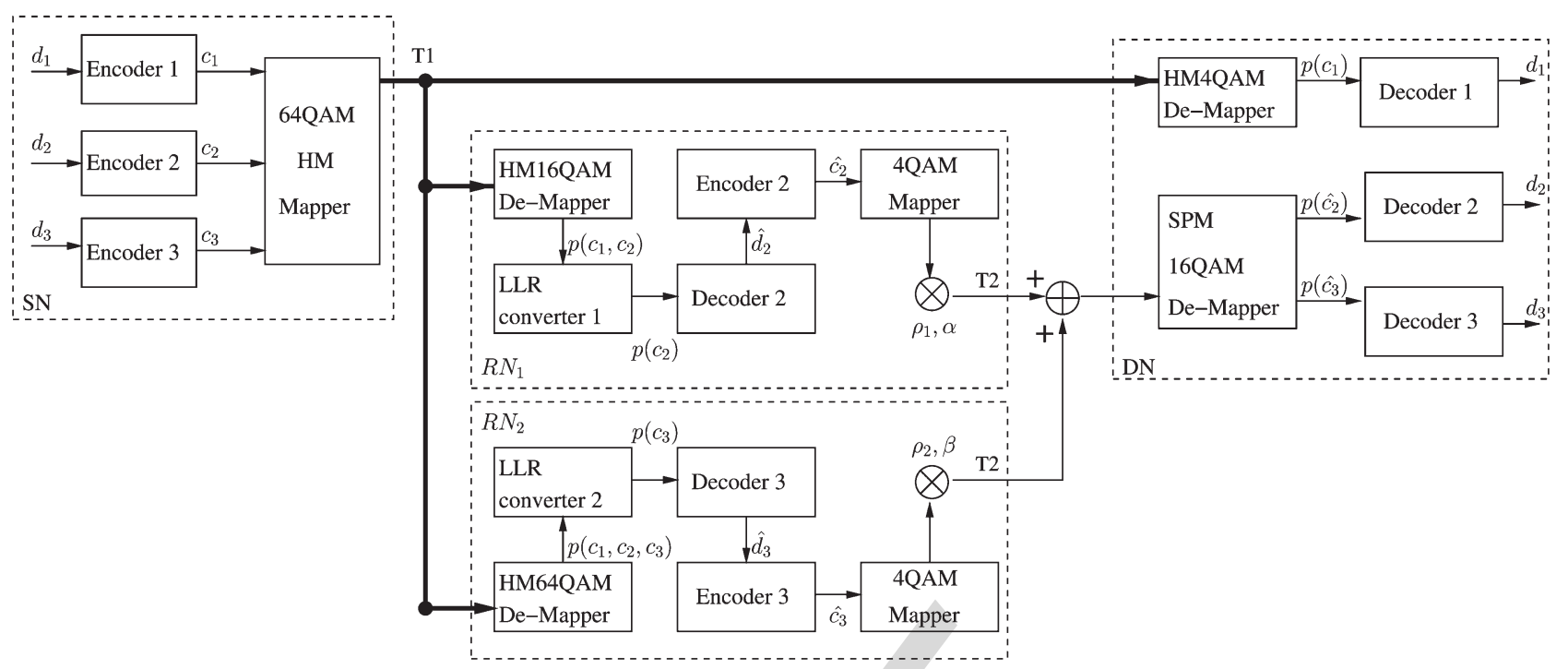

Fig. 2. The system diagram of a twin-relay HM and SPM aided cooperative system.

information contained in the other two layers would be decoded and retransmitted by the RNs. In order to reduce the complexity of the entire system, two RNs are activated for assisting the transmissions and each of the two RNs is used for retransmitting only one information layer of the triple-layer HM-64QAM symbols. With aid of the pre-coding and SPM schemes, the two RNs become capable of transmitting simultaneously. Hence, the system now needs two TSs to complete its transmissions between the SN and DN. More specifically, each RN will only deal with a single layer of the triple-layer HM-64QAM scheme, so the signal frames they transmit are all 4QAM symbol frames. In this way, both the processing complexity of the entire system and the transmit power of the $\mathrm{RN}$ is reduced.

\section{HM AND SPM MODULATION SCHEMES}

\section{A. Triple Layer HM Scheme}

Our triple-layer model of the HM-64QAM constellation seen in Fig. 3 was originally introduced in [26]. Since TTCM is used, where the symbol-based decoder's performance is determined by the Symbol Error Rate (SER), hence set-partition based mapping is invoked by the HM constellation instead of Gray mapping.

We define the six bits in a HM-64QAM symbol as $\left(b_{5} b_{4} b_{3} b_{2} b_{1} b_{0}\right)$, where the base layer or first layer $\left(L_{1}\right)$ is occupied by $\left(b_{5} b_{4}\right),\left(b_{3} b_{2}\right)$ belong to the second layer $\left(L_{2}\right)$ and $\left(b_{1} b_{0}\right)$ are contained in the third layer $\left(L_{3}\right)$. The generation rule of the triple-layer HM-64QAM symbols may be expressed as:

$$
S_{H M-64 Q A M}=\beta\left[S_{4 Q A M} \pm \sqrt{2} \delta_{1} e^{ \pm \frac{\pi}{4} j} \pm \sqrt{2} \delta_{2} e^{ \pm \frac{\pi}{4} j}\right] .
$$

The parameter $\beta$ is used for normalizing the average symbol energy to unity, which given by $\beta=1 / \sqrt{1+2 \delta_{1}^{2}+2 \delta_{2}^{2}}$. Furthermore, the ratios $R_{1}=d_{1} / d_{0}$ and $R_{2}=d_{3} / d_{2}$ are defined for controlling the shape of the HM-64QAM constellations, as shown in Fig. 3, where all the three parameters $\beta, \delta_{1}$ and $\delta_{2}$ will

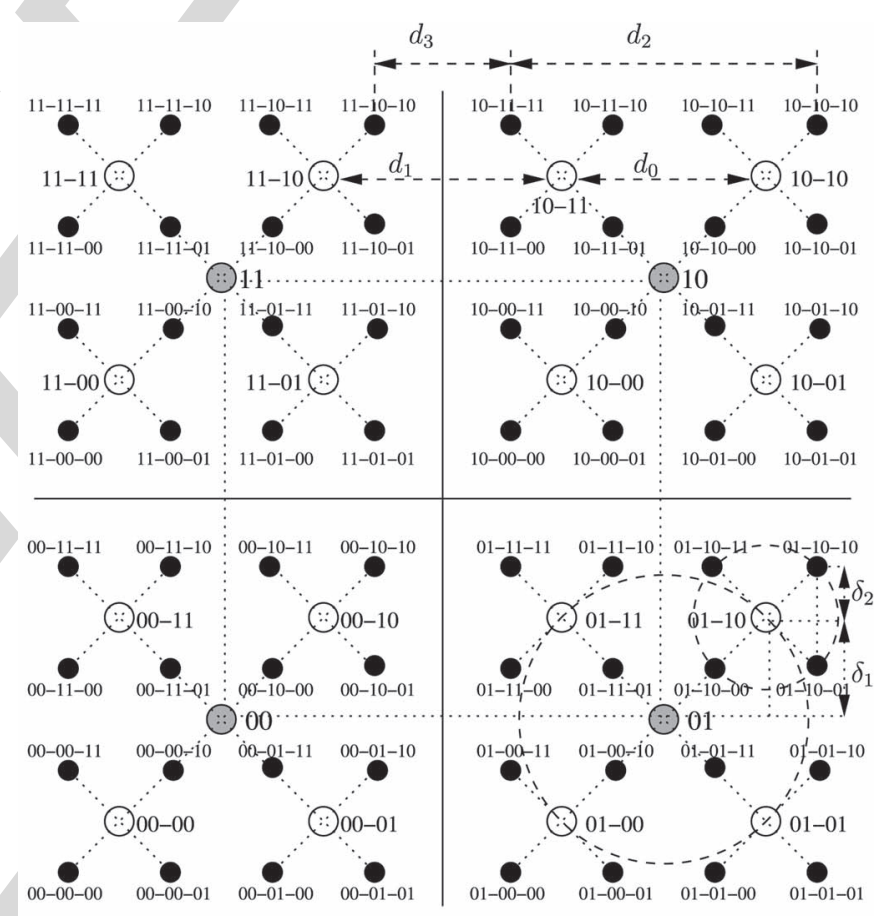

Base Layer: $b_{5} b_{4}$

HM 16QAM: $b_{5} b_{4}-b_{3} b_{2}$

HM 64QAM: $b_{5} b_{4}-b_{3} b_{2}-b_{1} b_{0}$

Fig. 3. The constellation map of the triple-layer HM-64QAM scheme, where $R_{1}=d_{1} / d_{0}, R_{2}=d_{3} / d_{2}$.

be directly controlled by the HM ratio pair $\left(R_{1}, R_{2}\right)$ and their relationship may be expressed as follows:

$$
\begin{aligned}
\delta_{1} & =\frac{1}{\sqrt{2}\left(1+R_{1}\right)}, \\
\delta_{2} & =\frac{R_{1}-R_{2}}{\sqrt{2}\left(1+R_{1}\right)\left(1+R_{2}\right)} .
\end{aligned}
$$


The constraint of the HM ratio pair $\left(R_{1}, R_{2}\right)$ in the simulations are:

$$
\begin{cases}0<R_{2}<R_{1} & \text { if } R_{1}<1 \\ \frac{1}{2}\left(R_{1}-1\right)<R_{2}<R_{1} & \text { if } R_{1}>1\end{cases}
$$

The derivations of (14), (15) and (16) are detailed in Appendices A, B, C and D respectively. The entire HM-64QAM constellation point arrangement is directly controlled by the HM ratios $R_{1}$ and $R_{2}$. Upon increasing the value of these two HM ratios, the constellation points in each quadrant would move closer to each other. Hence it is necessary to have a higher receive $\mathrm{SNR}$ at the $\mathrm{RN}\left(\mathrm{SNR}_{r}^{R N}\right)$ in order to adequately detect the information contained both in $L_{2}$ as well as in $L_{3}$, but a lower $\mathrm{SNR}_{r}^{D N}$ is necessitated for detecting the two bits in $L_{1}$. Our design-goal is to find the optimum $\mathrm{HM}$ ratios and RN position based on a given $\mathrm{SNR}_{t}^{S N}$.

\section{B. Twin Layer SPM Scheme}

The twin layer SPM scheme is detailed in [34], where we observe from this (9) that the reception of the signal at the DN is identical to that of detecting a twin-layer linear SPM signal received over AWGN channels. Note that the performance of a specific modulation scheme in the AWGN channel is directly determined by the Euclidean distance among the constellation points. The relationship between $\alpha$ and $\beta$ is given by [34]:

$$
\alpha^{2}+\beta^{2}=1
$$

If we only focus our attention on the relationship between the Euclidean distance $d_{\min }$ and $\alpha$, we have:

$d_{\min }=\left\{\begin{array}{ll}\frac{1}{\sqrt{2}} \min \left(2 \sqrt{1-\alpha^{2}}, 2\left(\alpha-\sqrt{1-\alpha^{2}}\right)\right) & (\alpha \geq \sqrt{1 / 2}) \\ \frac{1}{\sqrt{2}} \min \left(2 \alpha, 2\left(\sqrt{1-\alpha^{2}}-\alpha\right)\right) & (\alpha<\sqrt{1 / 2})\end{array}\right.$.

Theoretically the largest Euclidean distance is achieved, when $\alpha$ is $\sqrt{1 / 5}$ or $\sqrt{4 / 5}$. Hence, we anticipate that the best performance of an uncoded twin-layer linear SPM scheme would appear when $\alpha$ equals to $\sqrt{1 / 5}$ or $\sqrt{4 / 5}$. However, when TTCM is used, it depends on $d_{\min }$ of the entire TTCM set partitioning scheme [17].

\section{DEMAPPER AND RN POSITION}

In [34], we have discussed the receiving of the two signal frames using SPM schemes, so in this section, we only focus our attention on the receiving of the triple-layer TTCHM64QAM symbols. The symbol-to-bit Demapper block of Fig. 2 will produce a $(N \times M)$-element Probability Density Function (PDF) matrix of receiving $y$ given $x^{(i)}$ transmitted. $x^{(i)}$ is the hypothetically transmitted $M$-ray symbol for $i \in$ $\{0,1, \cdots, M-1\}$ and the element in the matrix is $p\left(y \mid x^{(i)}\right)$, which is the soft-input to the TTCM decoder, $N$ is the number of symbols in a transmission block. The general equation of calculating the PDF of receiving $y$, given that $x^{(i)}$ is transmitted may be expressed as:

$$
p\left(y \mid x^{(i)}\right)=\frac{1}{\pi N_{0}} \exp \left(\begin{array}{r}
\left.-\frac{\left|y-\sqrt{G} h x^{(i)}\right|^{2}}{N_{0}}\right) \\
\quad \times i \in\{0,1, \ldots, M-1\},
\end{array}\right.
$$

where $h$ is the fading coefficient and $G$ is the path-gain.

\section{A. $L_{1}$ Detection at $D N$}

The DN of Fig. 2 will demap the HM-64QAM signal frames received from the SN as 4QAM symbols for detecting the information contained in the base layer of the triple-layer HM-64QAM constellation. According to the HM-64QAM generation rule of (13), (19) may be rewritten as:

$$
\begin{gathered}
p\left(y_{S D} \mid x^{(i)}\right)=\frac{1}{\pi N_{0}} \exp \left(-\frac{\left|y_{S D}-\sqrt{G_{S D}} h_{S D} x^{(i)}\right|^{2}}{N_{0}}\right) \\
\times x^{(i)} \in\left\{\beta e^{j \pi / 4}, \beta e^{j 3 \pi / 4}, \beta e^{j-3 \pi / 4}, \beta e^{j-\pi / 4}\right\},
\end{gathered}
$$

where we have $i \in\{0,1,2,3\}$.

\section{B. $L_{2}$ Detection at $R N_{1}$}

The information output of Encoder 2 in Fig. 2 is mapped onto $L_{2}, R N_{1}$ will demap the received signal frame $x_{1}$ as the HM-16QAM symbols shown in Fig. 3 and will obtain the joint symbol probability of $L_{1}$ and $L_{2}$ in the HM-64QAM symbol streams for producing a $(N \times 16)$-element PDF matrix. Then, the Log Likelihood Ratio (LLR) converter 1 of Fig. 2, will extract the PDF of $L_{2}$ from the $(N \times 16)$-element PDF matrix. Therefore, $R N_{1}$ can decode $L_{2}$ even when $L_{1}$ is received with errors. When demapping the HM-64QAM symbol as HM-16QAM, (19) may be reformulated as:

$$
\begin{aligned}
p\left(y_{S R_{1}} \mid x^{(i)}\right)= & \frac{1}{\pi N_{0}} \exp \left(-\frac{\left|y_{S R_{1}}-\sqrt{G_{S R_{1}}} h_{S R_{1}} x^{(i)}\right|^{2}}{N_{0}}\right) \\
& \times x^{(i)} \in\left\{\beta\left[S_{4 Q A M} \pm \sqrt{2} \delta_{1} e^{ \pm \frac{\pi}{4} j}\right]\right\}, \quad(21)
\end{aligned}
$$

where $i \in\{0,1, \cdots, 15\}$. The HM-16QAM constellation points of $x^{(i)}$ are shown as hollow circles in Fig. 3. We defined $L_{2}^{(0)}$ as the pair of bits (00) in $L_{2}, L_{2}^{(1)}$ as $(01), L_{2}^{(2)}(10)$ and finally $L_{2}^{(3)}$ for (11), where the corresponding generation rule is given by:

$$
\begin{aligned}
p\left(y_{S R_{1}} \mid L_{2}^{(l)}\right) & =p\left(y_{S R_{1}} \mid x^{(l)}\right)+p\left(y_{S R_{1}} \mid x^{(l+4)}\right) \\
& +p\left(y_{S R_{1}} \mid x^{(l+8)}\right)+p\left(y_{S R_{1}} \mid x^{(l+12)}\right),
\end{aligned}
$$

where $l \in\{0,1,2,3\}$. The input PDF matrix of Decoder 2 is formulated in (22).

\section{C. $L_{3}$ Detection at $R N_{2}$}

$R N_{2}$ requires the highest receive power, because it has to convey the information of $L_{3}$. In order to receive $L_{3}, R N_{2}$ has 
to fully demap the whole HM-64QAM symbol stream. Hence (19) may be represented as:

$$
\begin{gathered}
p\left(y_{S R_{2}} \mid x^{(i)}\right)=\frac{1}{\pi N_{0}} \exp \left(-\frac{\left|y_{S R_{2}}-\sqrt{G_{S R_{2}}} h_{S R_{2}} x^{(i)}\right|^{2}}{N_{0}}\right) \\
\times x^{(i)} \in\left\{\beta\left[S_{4 Q A M} \pm \sqrt{2} \delta_{1} e^{ \pm \frac{\pi}{4} j} \pm \sqrt{2} \delta_{2} e^{ \pm \frac{\pi}{4} j}\right]\right\},
\end{gathered}
$$

where $i \in\{0,1, \cdots, 63\}$. Let $L_{3}^{(0)}$ denote the pair of bits (00) in $L_{3}, L_{3}^{(1)}$ represent (01), $L_{2}^{(3)}(10)$ and finally $L_{3}^{(3)}$ for (11). Then the LLR converter 2 of Fig. 2 may produce the PDF of $L_{3}$ according to:

$$
p\left(y_{S R_{2}} \mid L_{3}^{(l)}\right)=\sum_{k=0}^{15} p\left(y_{S R_{1}} \mid x^{(i=4 k+l)}\right),
$$

where $l \in\{0,1,2,3\}$. The $(N \times 4)$-element PDF matrix generated by the LLR converter 2 is then fed to Decoder 3 of $\mathrm{RN}_{2}$, as seen in Fig. 2, for detecting $L_{3}$. Furthermore, we consider logarithmic probabilities, so that the approximate log MAP algorithm [17] may be directly employed by the decoder block.

\section{RN Position}

In our simulations, the same rate- $1 / 2$ encoder is employed for all three SN encoders. Hence we only focus our attention on the specific SNR values for achieving a BER of $10^{-6}$. Multiple values of the two HM ratios $R_{1}$ and $R_{2}$ had been tested. At a given $\mathrm{HM}$ constellation ratio pair $\left(R_{1}, R_{2}\right)$, the minimum receive SNR required $S N R_{r}^{L_{1}}$ for decoding $L_{1}$ at the DN, $S N R_{r}^{L_{2}}$ for receive $L_{2}$ at the $R N_{1}$ and $S N R_{r}^{L_{3}}$ for receiving $L_{3}$ at $R N_{2}$ may be computed. The SNR differences among the three layers are:

$$
\begin{aligned}
& \mathcal{G}_{S N R}^{L_{1}, L_{2}}=S N R_{r}^{L_{2}}-S N R_{r}^{L_{1}}[\mathrm{~dB}], \\
& \mathcal{G}_{S N R}^{L_{1}, L_{3}}=S N R_{r}^{L_{3}}-S N R_{r}^{L_{1}}[\mathrm{~dB}],
\end{aligned}
$$

where, $\mathcal{G}_{S N R}^{L_{1}, L_{j}}$ is the SNR difference between $S N R_{r}^{L_{1}}$ and $S N R_{r}^{L_{j}}$, for $j \in\{2,3\}$. If we set $\operatorname{SNR}_{t}^{S N}$ to be identical to the SNR required for receiving $L_{1}$ from the HM-64QAM symbol, namely to $S N R_{t}^{S N}=S N R_{r}^{L_{1}}$, this would guarantee that the BER of decoding $L_{1}$ would reach an arbitrarily low value. In this situation, if we want the BER performance of receiving $L_{2}$ to become sufficiently low, the channel gain $G_{S R_{1}}$ of the $\mathrm{SN}-\mathrm{RN}_{1}$ link should satisfy:

$$
10 \log _{10} G_{S R_{1}}+S N R_{r}^{L_{1}}=S N R_{r}^{L_{2}} .
$$

If we use the distance-ratio $d_{S R_{1}} / d_{S D}$ to represent the position of the RN, we arrive at:

$$
\mathcal{G}_{S N R}^{L_{1}, L_{2}}=10 \log _{10}\left(\frac{d_{S D}}{d_{S R_{1}}}\right)^{3}
$$

where $\mathcal{G}_{S N R}^{L_{1}, L_{2}}$ is given by (25) and hence we have:

$$
\frac{d_{S R_{1}}}{d_{S D}}=10^{-\frac{\mathcal{G}_{S N R}^{L_{1} L_{2}}}{30}} .
$$

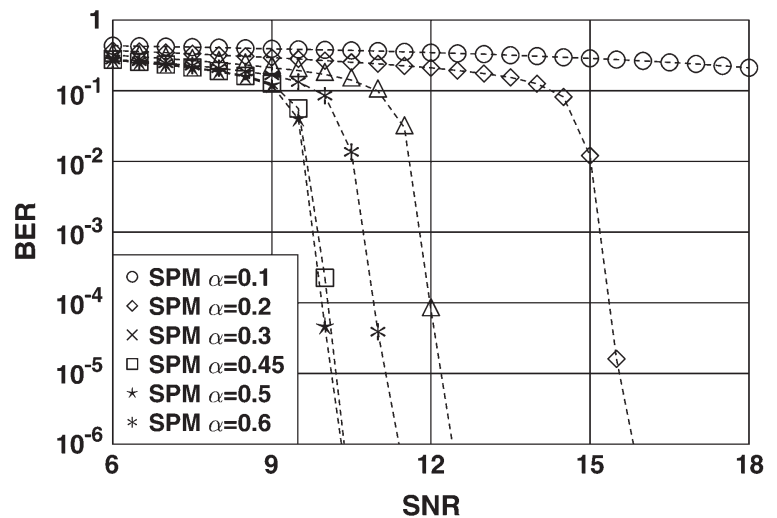

(a)

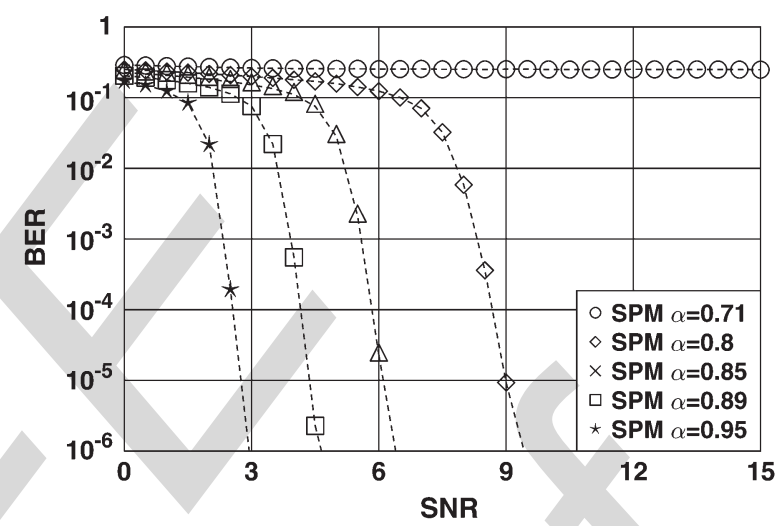

(b)

Fig. 4. The BER versus SNR performance of receiving a single signal sequence with SPM ratio $\alpha$ in the twin layer SPM schemes. Both of the two signal sequences are encoded by rate-1/2 TTCM encoder. The number of iterations of the rate-1/2 TTCM decoder is $\zeta=4$, the block size is $\eta=12,000$ symbols and the channel is uncorrelated Rayleigh fading channel.

Similarly, the position of $\mathrm{RN}_{2}$ is related to:

$$
\frac{d_{S R_{2}}}{d_{S D}}=10^{-\frac{\mathcal{G}_{S N R}^{L_{1}, L_{3}}}{30}} .
$$

\section{TRIPLE-LAYER TTCHM-64QAM CoOPERATIVE SYSTEM DESIGN}

In practice we do not have any control over the position of mobile relays, but the relay-selection algorithm would appoint a relay close to the optimum location. In this section, we will optimize this practical system regarding the position of the RN, as well as the HM ratio pair $\left(R_{1}, R_{2}\right)$ and the SPM weighting pair $(\alpha, \beta)$. Additionally, in this investigation, the simulations are carried out by IT++and the number of iterations of our rate-1/2 TTCM decoder is $\zeta=4$, the block size is $\eta=12,000$ symbols. Using a large number of iterations allows the TTCM decoder to more closely approach capacity and a large block length assists in avoiding error propagation, but also imposes an increased complexity. In the simulations, we observed that no substantial BER performance improvement is achieved for more than four iterations $(\zeta>4)$ or for a block size of $\eta>$ 12,000 symbols. 


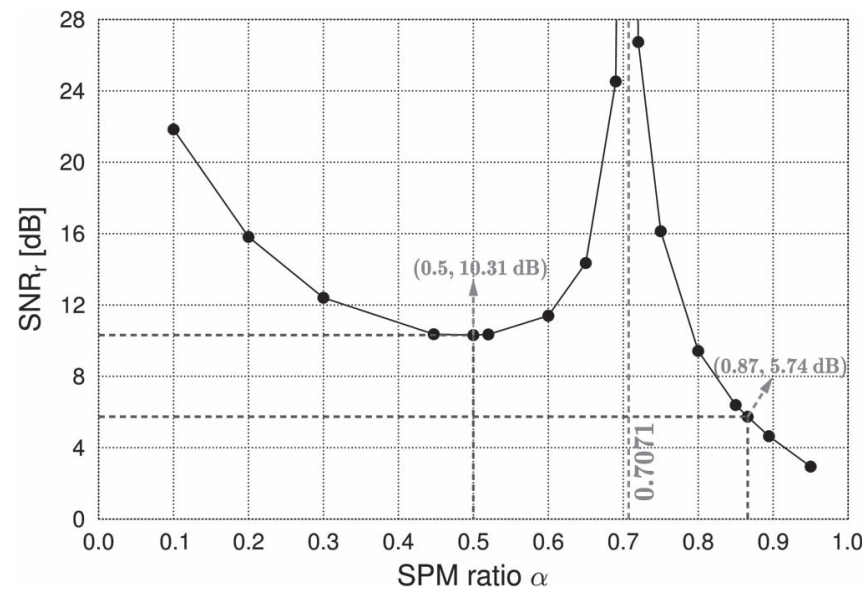

Fig. 5. The $\mathrm{SNR}_{r}$ versus SPM ratio performance of receiving a single signal sequence with SPM ratio $\alpha$ in the twin layer SPM schemes. Both of the two signal sequences are encoded by rate- $1 / 2$ TTCM encoder. The number of iterations of the rate- $1 / 2$ TTCM decoder is $\zeta=4$, the block size is $\eta=$ 12,000 symbols and the channel is uncorrelated Rayleigh fading channel. The $\mathrm{SNR}_{r}$ here is the required receive $\mathrm{SNR} r$ for achieving a performance of $\mathrm{BER}=10^{-6}$.

\section{A. Optimum SPM Ratio}

In our simulations, the pair of signal sequences received from $\mathrm{RN}_{1}$ and $\mathrm{RN}_{2}$ will be multiplied by a specific SPM ratio $\alpha$ or $\beta$ and be received by the DN simultaneously. Here, we only focus our attention on the performance of receiving a single signal sequence having a SPM ratio $\alpha$ in the simulations and the related results are shown in Fig. 4(a) and Fig. 4(b).

From the combined signal sequences, we set the signal associated with a larger SPM ratio $\alpha$ to be the dominant signal and the signal with a smaller SPM ratio $\beta$ to be the auxiliary signal. Based on the simulation results of Fig. 4(a) and Fig. 4(b), we may observe that as expected, for the dominant signal, a larger SPM ratio would result in a better BER performance. However, for the auxiliary signal, the best BER performance appears at the SPM ratio of $0.45(\sqrt{1 / 5} \approx 0.45)$ or 0.5 . We have found from our simulations the $\mathrm{SNR}_{r}$ value required for achieving $\mathrm{BER}=10^{-6}$ for different values of the SPM ratio $\alpha$, as shown in Fig. 5. Note that $0.7071(\sqrt{1 / 2} \approx 0.7071)$ is a bound, where we have $\alpha=\beta=\sqrt{1 / 2}$, which would collapse the 16 -point constellation to a 9-point constellation making unambiguous decoding to be impossible. Fig. 5 shows that when $\alpha=0.5$, the $\mathrm{SNR}_{r}$ required for receiving the auxiliary signal from the SPM16QAM signal is about $10.31 \mathrm{~dB}$ which is the lowest value of receiving the auxiliary signal. Meanwhile, when $\alpha=0.5$, the corresponding SPM ratio $\beta$ will be $\sqrt{1-\alpha^{2}} \approx 0.87$, and the $\mathrm{SNR}_{r}$ required for receiving the dominant signal from the SPM16QAM signal using $\beta=0.87$ is about $5.74 \mathrm{~dB}$. Hence, based on the results of Fig. 5, we find that the optimum SPM ratio pair is ( $\alpha=0.87, \beta=0.5)$ (we assume $\alpha>\beta$ in this paper). In this combination, the $\mathrm{SNR}_{r}$ required for receiving the two signal sequences is the lowest. Consequently, the $\mathrm{SNR}_{t}$ required at the two RNs would also be the lowest at the given RN positions.

\section{B. SNR of the Two RNs}

In order to evaluate the power-efficiency of our cooperative communications scheme, we have to calculate the average
$\mathrm{SNR}_{t}\left(\overline{S N R_{t}}\right)$ defined as the $\mathrm{SNR}_{t}$ per TS. Based on the statistics seen in Fig. 5, we opted for the SPM ratio pair of ( $\alpha=$ $0.87, \beta=0.5)$ and hence we have $\operatorname{SNR}_{r}^{D N}=10.31 \mathrm{~dB}$. Next, we have to find the relationship among $\mathrm{SNR}_{t}^{R N_{1}}, \mathrm{SNR}_{t}^{R N_{2}}$ and $\operatorname{SNR}_{r}^{D N}$. Let us denote the SNR by $\gamma$, which is expressed as $10 \log _{10}(\gamma)$ in $\mathrm{dB}$. Furthermore, since $L_{2}$ of the triple-layer HM-64QAM symbols has a higher priority than that of $L_{3}$, the signal frame $\left\{x_{2}\right\}$ transmitted from $\mathrm{RN}_{1}$ will be multiplied by a higher SPM weighting factor of $\alpha=0.87$, while another SPM weighting factor of $\beta=0.5$ is used for the signal frame $\left\{x_{3}\right\}$ at $\mathrm{RN}_{2}$. Hence we have:

$$
E\left[\gamma_{t}^{R N_{1}}\right]=E\left[\frac{\left|\alpha \rho_{1} x_{2}\right|^{2}}{N_{0}}\right]
$$

where

$$
E\left[\left|x_{2}\right|^{2}\right]=1
$$

Therefore, it can be observed that:

$$
\begin{aligned}
E\left[\gamma_{t}^{R N_{1}}\right] & =E\left[\frac{\alpha^{2}\left|\rho_{1}\right|^{2}}{N_{0}}\right] \\
& =\alpha^{2} E\left[\frac{\left|h_{R_{1} D}^{*}\right|^{2}}{\left|h_{R_{1} D}\right|^{4} G_{R_{1} D} N_{0}}\right] \\
& =\frac{\alpha^{2}}{G_{R_{1} D} N_{0}} E\left[\frac{1}{\left|h_{R_{1} D}\right|^{2}}\right],
\end{aligned}
$$

where $h$ obeys the Rayleigh distribution of [32]:

$$
f(h)=\frac{2 h}{\Omega} \exp \left(-\frac{h^{2}}{\Omega}\right) .
$$

Note that the mean square value of $h$ is given by $\Omega=1$, Let $Z=|h|^{2}$, then the distribution of the variable $Z$ may be expressed as [32]:

$$
f_{Z}(z)=\frac{1}{\Omega} \exp \left(-\frac{z}{\Omega}\right) .
$$

Let us denote the Cumulative Distribution Function (CDF) as $F_{Z}(\Gamma)=\operatorname{Pr}(Z<\Gamma)$, which can be expressed as:

$$
F_{Z}(\Gamma)=\operatorname{Pr}(Z<\Gamma)=1-\exp \left(-\frac{\Gamma}{\Omega}\right),
$$

where $\operatorname{Pr}$ denotes probability. Upon introducing $\Gamma^{\prime}=1 / \Gamma$, we may further express (36) as:

$$
\operatorname{Pr}(Z<\Gamma)=\operatorname{Pr}\left(\frac{1}{Z}>\frac{1}{\Gamma}\right)=1-\operatorname{Pr}\left(\frac{1}{Z}<\Gamma^{\prime}\right) .
$$

Hence, it can be observed that:

$$
\operatorname{Pr}\left(\frac{1}{Z}<\Gamma^{\prime}\right)=\exp \left(-\frac{1}{\Omega \Gamma^{\prime}}\right) \text {. }
$$

If we let $\Theta=\frac{1}{Z}$, the PDF of the variable $\Theta$ may be expressed as [35]:

$$
f_{\Theta}(\theta)=\frac{1}{\Omega \theta^{2}} \exp \left(-\frac{1}{\Omega \theta}\right)
$$


Therefore, the expectation $E\left[\frac{1}{|h|^{2}}\right]$ may be derived as:

$$
E\left[\frac{1}{|h|^{2}}\right]=\int_{\Theta_{\text {low }}}^{\Theta_{u p}} \frac{\Theta}{\Omega \Theta^{2}} \exp \left(-\frac{1}{\Omega \Theta}\right) \mathrm{d} \Theta,
$$

where $\Theta_{\text {low }}$ and $\Theta_{\text {up }}$ are the lower and upper limits of the integration. Let us define $\xi=\frac{1}{\Theta}$, then (40) may be converted to:

$$
\frac{1}{\Omega} \int_{\frac{1}{\Theta_{u p}}}^{\frac{1}{\Theta_{\text {low }}}} \frac{1}{\xi} \exp \left(-\frac{\xi}{\Omega}\right) \mathrm{d} \xi=\frac{1}{\Omega}\left[E_{i}\left(\frac{-1}{\Omega \Theta_{u p}}\right)-E_{i}\left(\frac{-1}{\Omega \Theta_{\text {low }}}\right)\right] \text {, }
$$

where $E_{i}$ is the Euler function:

$$
E_{i}(u)=\int_{-u}^{\infty} \frac{e^{-t}}{t} \mathrm{~d} t
$$

Theoretically, we have $|h| \in[0,+\infty)$, which gives us $\Theta_{\text {low }}=0$ and $\Theta_{\text {up }}=+\infty$. When $\Theta_{\text {low }}=0$, we may have $E_{i}\left(\frac{-1}{\Omega \Theta_{\text {low }}}\right)=0$. However, if $\Theta_{u p}=+\infty$, the term $E_{i}\left(\frac{-1}{\Omega \Theta_{u p}}\right)$ becomes infinite and we are unable to derive the value of $E\left[1 /|h|^{2}\right]$. To resolve this dilemma, we defined an outage threshold, which is given by $\left[|h|^{2}\right]_{\min }=0.03$. According to (35), the probability of $\operatorname{Pr}\left(|h|^{2} \geqslant 0.03\right) \approx 0.97$, indicates that our system will halt its transmissions, when the fading obeys $|h|^{2}<0.03$. Hence, we may have $\frac{1}{\Theta_{u p}}=\left[|h|^{2}\right]_{\min }=0.03$. In this situation, we have $E\left[1 /|h|^{2}\right]=2.96$. Hence, (33) may be expressed as:

$$
E\left[\gamma_{t}^{R N_{1}}\right]=\frac{2.96 \alpha^{2}}{G_{R_{1} D} N_{0}}
$$

Note that this assumption will lead to a $3 \%$ throughput reduction for the entire system. Additionally, we have:

$$
E\left[\gamma_{r}^{D N}\right]=E\left[\frac{\left|\alpha x_{2}+\beta x_{3}\right|^{2}}{N_{0}}\right]=\frac{1}{N_{0}} .
$$

Therefore, we find that:

$$
E\left[\gamma_{t}^{R N_{1}}\right]=\frac{2.96 \alpha^{2}}{G_{R_{1} D}} E\left[\gamma_{r}^{D N}\right]
$$

which may be expressed in $\mathrm{dB}$ as:

$$
E\left[S N R_{t}^{R N_{1}}\right]=E\left[S N R_{r}^{D N}\right]+10 \log _{10}\left(\frac{2.96 \alpha^{2}}{G_{R_{1} D}}\right) .
$$

Similarly, the relationship between $S N R_{t}^{R N_{2}}$ and $S N R_{r}^{D N}$ may be formulated as:

$$
E\left[S N R_{t}^{R N_{2}}\right]=E\left[S N R_{r}^{D N}\right]+10 \log _{10}\left(\frac{2.96 \beta^{2}}{G_{R_{2} D}}\right) .
$$

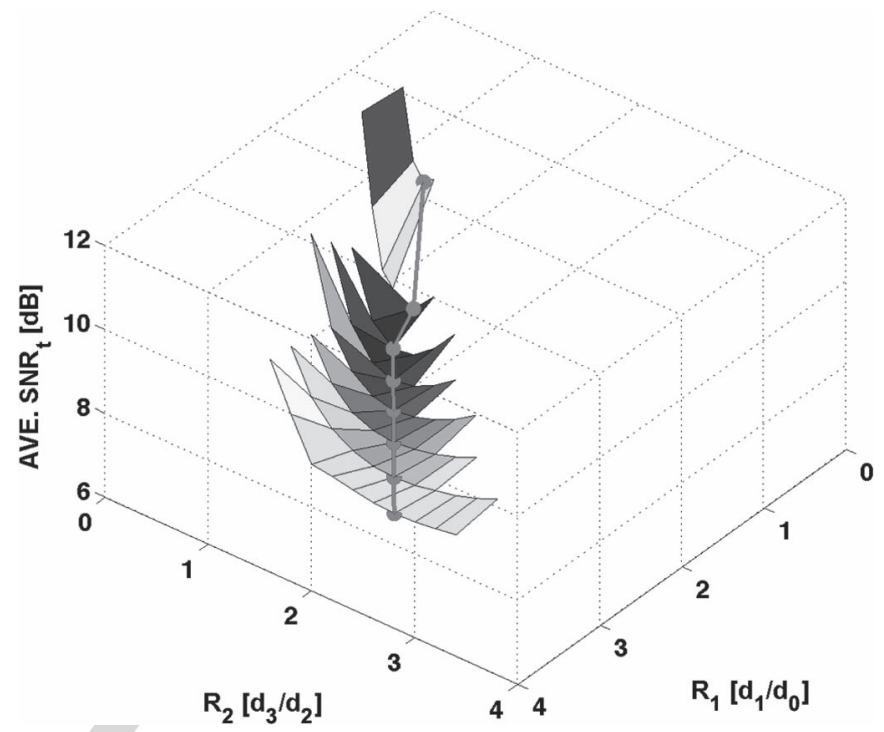

Fig. 6. The 3D plot of the simulation-based transmit power dissipation surface of the entire system versus the HM-64QAM ratio pair $\left(R_{1}, R_{2}\right)$. The two RNs employ SPM schemes associated with the SPM weighting pair of $(\alpha=0.87$, $\beta=0.5$ ). Explicitly, the number of iterations of the rate-1/2 TTCM decoder is $\zeta=4$, the block size is $\eta=12,000$ symbols and an uncorrelated Rayleigh fading channel is considered.

Finally, $\overline{S N R_{t}}$ may be expressed as:

$\overline{S N R_{t}}=10 \log _{10}\left(\overline{\gamma_{t}}\right)=10 \log _{10}\left(\frac{\gamma_{t}^{S N}+\gamma_{t}^{R N_{1}}+\gamma_{t}^{R N_{2}}}{2}\right)$.

\section{Simulation Results}

Based on Section V, we characterized our cooperative communications system for multiple values of the HM ratio pairs $\left(R_{1}, R_{2}\right)$ for the sake of generating the power dissipation surface of the cooperative system, which is shown in Fig. 6. Explicitly, 64 pairs of $\left(R_{1}, R_{2}\right)$ have been simulated, where $R_{1}$ is chosen from $\{0.5,1.0,1.5,2.0,2.5,3.0,3.5,4.0\}$ and for each $R_{1}$, multiple $R_{2}$ values were selected based on the constraints illustrated in Section III. Given a specific HM ratio pair $\left(R_{1}, R_{2}\right)$, the receive SNR required for adequately receiving $L_{1}, L_{2}$ and $L_{3}$, namely $\mathrm{SNR}_{r}^{L_{1}}, \mathrm{SNR}_{r}^{L_{2}}$ and $\mathrm{SNR}_{r}^{L_{3}}$ respectively, may be derived for a target BER of $10^{-6}$. More specifically, the optimum position of the two RNs and the optimum average transmit $\operatorname{SNR}\left(\overline{S N R_{t}}\right)$ of the entire system may be calculated according to $\mathrm{SNR}_{r}^{L_{1}}, \mathrm{SNR}_{r}^{L_{2}}$ and $\mathrm{SNR}_{r}^{L_{3}}$ based on the discussions in Sections IV and Section V. Hence, we can compute the optimum $\overline{S N R_{t}}$ for each of the HM ratio pairs $\left(R_{1}, R_{2}\right)$, as shown in Fig. 6 . The bold line marked by dots in Fig. 6 illustrates the lowest power consumption point for a specific $\mathrm{HM}$ ratio pair $\left(R_{1}, R_{2}\right)$ and the corresponding data is recorded in Table I.

Based on the results of Fig. 6, the best performance of our cooperative communication system obeying this arrangement is achieved, when the HM ratio pair is given by $\left(R_{1}=1.5\right.$, $R_{2}=0.8$ ) and the SPM weighting factor pair is ( $\alpha=0.87, \beta=$ 0.5 ), where the optimum $\overline{S N R_{t}}$ per TS is $6.94 \mathrm{~dB}$. In this situation, the positions of $\mathrm{RN}_{1}$ and $\mathrm{RN}_{2}$ are $d_{S R_{1}} / d_{S D}=0.53$ and $d_{S R_{2}} / d_{S D}=0.31$, where $\mathrm{SNR}_{t}^{S N}$ is $6.81 \mathrm{~dB}, \mathrm{SNR}_{t}^{R N_{1}}$ is 
TABLE I

The Simulation Based Average Transmission Power $\overline{S N R_{t}}$ Per TS of the HM-64QAM and SPM Based Cooperative System. The SPM WeIghting PAIR IS $(\alpha=0.87, \beta=0.5)$, THE NUMBER OF ITERATIONS OF THE RATE- $1 / 2$ TTCM DECODER IS $\zeta=4$, AND THE BLOCK SizE

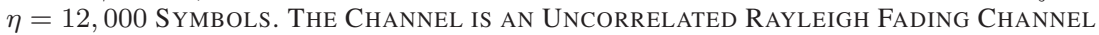

\begin{tabular}{|l|l|l|l|l|l|l|l|}
\hline$R_{1}$ & $R_{2}$ & $\begin{array}{l}\text { Pos. } \\
{\left[d_{S R_{1}} / d_{S D}\right]}\end{array}$ & $\begin{array}{l}\text { Pos. } \\
{\left[d_{S R_{2}} / d_{S D}\right]}\end{array}$ & $\begin{array}{l}S N R_{t}^{S N} \\
{[\mathrm{~dB}]}\end{array}$ & $\begin{array}{l}S N R_{t}^{R N_{1}} \\
{[\mathrm{~dB}]}\end{array}$ & $\begin{array}{l}S N R_{t}^{R N_{2}} \\
{[\mathrm{~dB}]}\end{array}$ & $\begin{array}{l}S N R_{t} \\
{[\mathrm{~dB}]}\end{array}$ \\
\hline 0.5 & 0.3 & 0.82 & 0.33 & 11.18 & -8.81 & 3.88 & 8.94 \\
\hline 1.0 & 0.6 & 0.65 & 0.30 & 7.98 & -0.04 & 4.27 & 6.96 \\
\hline 1.5 & 0.8 & 0.53 & 0.31 & 6.81 & 4.01 & 4.08 & $\mathbf{6 . 9 4}$ \\
\hline 2.0 & 1.2 & 0.47 & 0.26 & 5.91 & 5.61 & 5.12 & 7.32 \\
\hline 2.5 & 1.6 & 0.42 & 0.22 & 5.42 & 6.71 & 5.76 & 7.76 \\
\hline 3.0 & 2.0 & 0.38 & 0.19 & 5.06 & 7.52 & 6.20 & 8.14 \\
\hline 3.5 & 2.4 & 0.35 & 0.17 & 4.79 & 8.10 & 6.50 & 8.46 \\
\hline 4.0 & 2.8 & 0.33 & 0.16 & & 6.73 & 8.74 \\
\hline
\end{tabular}

$4.01 \mathrm{~dB}$ and $\mathrm{SNR}_{t}^{R N_{2}}$ is $4.08 \mathrm{~dB}$, as shown in Table I. The throughput per TS for this scheme is $3 / 2 \times 0.97=1.455 \mathrm{bps}$ owing to the $3 \%$ throughput reduction imposed by the threshold of $\left[|h|^{2}\right]_{\min }=0.03$.

\section{A. EXIT Chart Analysis}

The Extrinsic Information Transfer (EXIT) chart is capable of visualizing the input/output characteristics of the constituent MAP decoders in terms of the achievable average mutual information transfer [36], [37]. It may be used for predicting whether a soft decision based decoder is capable of decoding the information with an arbitrarily low BER based on the available mutual information provided by the symbol-to-bit demapper. Since we do not invoke iterations between the demapper and the TTCM decoder in our symbol-based scheme, we need a sufficiently high receive $\mathrm{SNR}_{r}$ for guaranteeing that the mutual information gleaned from the demapper is sufficiently high for the decoder to attain a low BER. More specifically, the EXIT curves of the HM-64QAM and SPM-16QAM demappers are illustrated in Fig. 7.

In Fig. 7, the notation 'Inner' iteration represents the information exchange between the demapper and decoder, while 'Outer' iteration refers to the information exchange between the two components of the TTCM decoder. Since there are no iterations between the demapper and the TTCM decoder, we may observe in Fig. 7 that the inner curve is a straight line. Specifically, the inner curve shows the mutual information received by the decoder from the demapper, which is increased upon increasing the receive SNR. In order to guarantee that the decoder becomes capable of decoding the information with an arbitrarily low BER, an open EXIT chart tunnel has to be maintained between the 'Inner' and 'Outer' curves all the way to the $(x, y)=(2, y)$ point, where we have $x=I_{A}^{(i)}=I_{E}^{(o)}$ and $y=I_{E}^{(i)}=I_{A}^{(o)}$. Note that the subscript $i$ and $o$ denote 'Inner' and 'Outer' respectively, whilst $I_{A}$ and $I_{E}$ denote the a priori and extrinsic information. When the HM-64QAM ratio pair is ( $R_{1}=1.5, R_{2}=0.8$ ) and the number of TTCM iterations is $\zeta=4$, the receive SNR required for achieving a BER of $10^{-6}$ for each HM layer is: $\mathrm{SNR}_{r}^{L_{1}}=6.81 \mathrm{~dB}, \mathrm{SNR}_{r}^{L_{2}}=15.15 \mathrm{~dB}$ and $\mathrm{SNR}_{r}^{L_{3}}=21.87 \mathrm{~dB}$. It also can be observed in Fig. 7 that there are open tunnels between the three 'Inner' curves upon receiving triple-layer HM symbols and the $\zeta=4$-iteration 'Outer' curve. Note that the 'Inner' curve of receiving $L_{3}$ of the

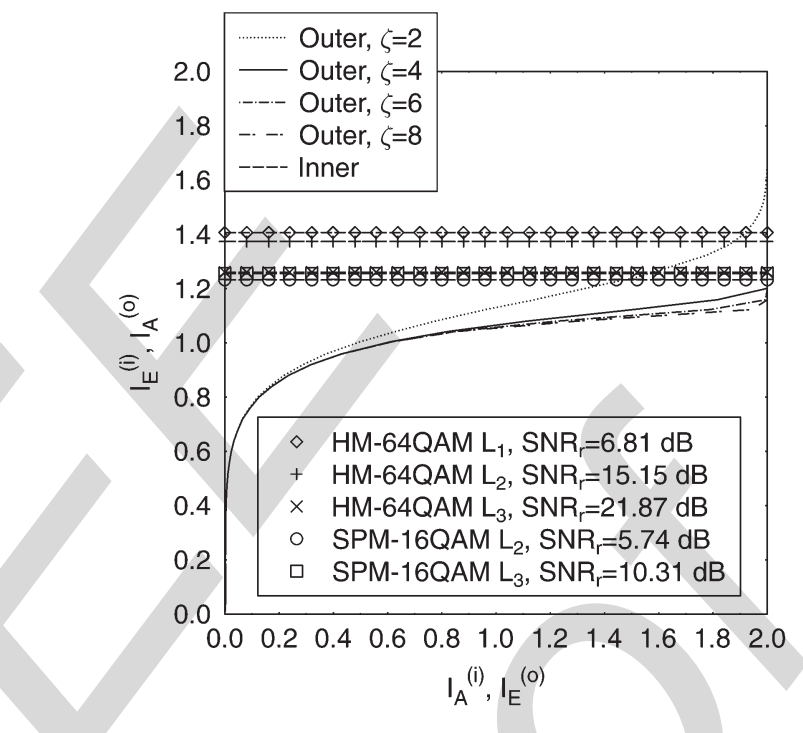

Fig. 7. The symbol based EXIT chart of our rate-1/2 TTCM aided triplelayer HM-64QAM scheme and twin-layer SPM-16QAM scheme. The number of iterations in the rate-1/2 TTCM decoder is from the set of $\zeta \in\{2,4,6,8\}$. The HM-64QAM ratio pair is $\left(R_{1}=1.5, R_{2}=0.8\right)$ and the SPM weighting coefficient pair is $(\alpha=0.87, \beta=0.5)$. The receive SNR required for achieving a BER of $10^{-6}$ based on simulations is denoted as $\mathrm{SNR}_{r}$. An uncorrelated Rayleigh fading channel is considered.

HM scheme is closer to the 'Outer' curve compared to the other two 'Inner' curves of receiving $L_{1}$ and $L_{2}$. When $L_{1}$ of the HM scheme is detected at the DN, each HM-64QAM symbol received will be detected as a 4QAM symbol. Similarly, $L_{2}$ of the HM scheme will be detected in the form of 16QAM symbols at $\mathrm{RN}_{1}$. This HM-specific simplifying demapping assumption shifts the 'Inner' curves corresponding to $L_{1}$ and $L_{2}$ upwards to higher values than the 'Inner' curve recorded for receiving $L_{3}$. Hence, we infer that we can reduce the SNR required for receiving $L_{1}$ and $L_{2}$ to let the two 'Inner' curves to be closer to the $\zeta=4$-iteration based 'Outer' curve. However, according to the simulations, even though there might be an open EXIT tunnel, the integrity of $L_{1}$ and $L_{2}$ will be degraded, if we reduce $\mathrm{SNR}_{r}$.

Additionally, the 'Inner' curves associated with receiving the pre-coding based twin-layer SPM-16QAM scheme are also shown in Fig. 7. In the simulations, the $L_{2}$ and $L_{3}$ of the triplelayer HM-64QAM will be mapped to base layer and auxiliary layer of the SPM symbols, respectively. It can be observed in Fig. 7 that the $\mathrm{SNR}_{r}$ required for achieving a BER of $10^{-6}$ for both layers of our twin-layer SPM scheme can provide open 


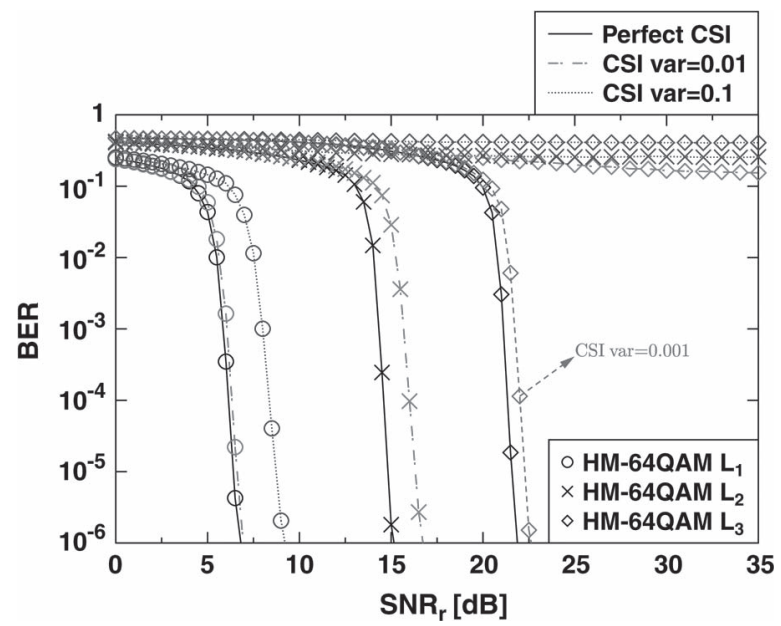

Fig. 8. BER versus SNR performance of the triple-layer coded HM-64QAM scheme with imperfect CSI, where the HM-64QAM ratio pair is $\left(R_{1}=\right.$ $\left.1.5, R_{2}=0.8\right)$. Explicitly, the number of iterations of the rate-1/2 TTCM decoder is $\zeta=4$, the block size is $\eta=12,000$ symbols and an uncorrelated Rayleigh fading channel is considered.

tunnels between the two 'Inner' curves and the $\zeta=4$-iteration 'Outer' curve. Moreover, both 'Inner' curves are very close to the $\zeta=4$-iteration 'Outer' curve, since the DN fully detects the SPM-16QAM symbols for the sake of receiving the softinformation of $L_{2}$ and $L_{3}$. We have investigated the optimum number of iterations for the TTCM decoder. It can be observed from Fig. 7 that increasing $\zeta$ beyond 4 only gives us a marginal gain, while significantly increasing the decoding complexity. Hence we have opted for $\zeta=4$ for our design.

\section{B. Imperfect CSI for Receiving Triple-Layer HM and Pre-Coding Based Twin-Layer SPM}

When considering the impact of imperfect CSI at all nodes in cooperative communications, the performance of our coherent scheme is expected to be degraded. To investigate the robustness of our triple-layer coded HM-64QAM and pre-coding based twin-layer coded SPM-16QAM schemes, we model the CSI estimation errors by a Gaussian process superimposed on each channel coefficient at the two RNs and DN, where the CSI estimation error variance is denoted by $\widetilde{\sigma}$.

The BER performance of receiving triple-layer coded HM-64QAM recorded for diverse CSI estimation error variances $\widetilde{\sigma}$ is shown in Fig. 8. We can observe that a CSI estimation error of $\widetilde{\sigma}=0.01$ only slightly impedes the performance of receiving $L_{1}$, but will cause a $2 \mathrm{~dB}$ SNR degradation (at a BER of $10^{-6}$ ) for receiving $L_{2}$. However, the system excessively degrade $L_{3}$ for $\widetilde{\sigma}=0.01$. By contrast, a CSI estimation error of $\tilde{\sigma}=0.1$ would impose a $5 \mathrm{~dB}$ SNR degradation on the performance of $L_{1}$, whilst the cooperative communication system will have completely lost $L_{2}$ and $L_{3}$ in this situation. It is shown in Fig. 8 that a CSI estimation error variance below $\widetilde{\sigma}=0.001$ is required for receiving $L_{3}$. Hence, we find that the robustness of each layer in the HM-64QAM symbols against imperfect CSI is different, where $L_{1}$ is the most robust layer, while $L_{3}$ has the highest sensitivity to CSI errors.

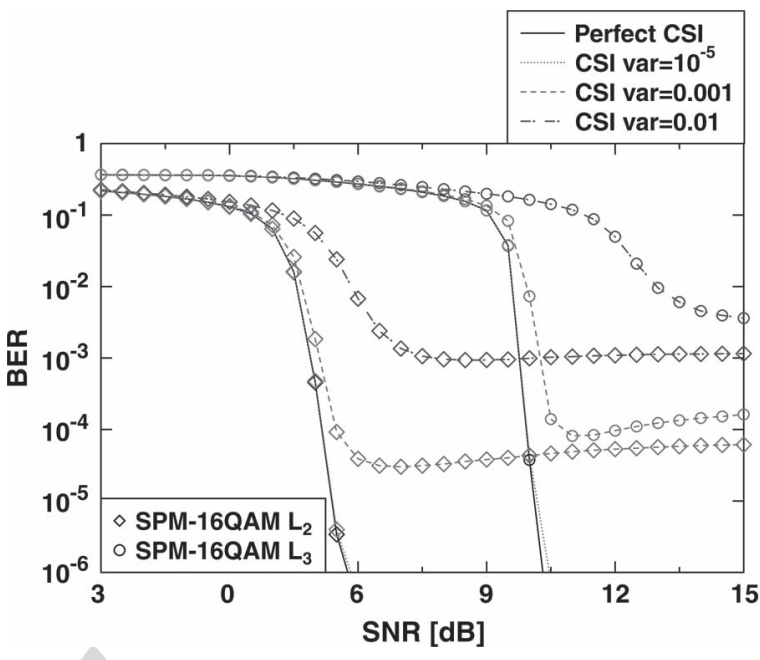

Fig. 9. BER versus SNR performance of the twin-layer coded SPM-16QAM scheme with imperfect CSI, where the SPM-16QAM weighting pair is $(\alpha=$ 0.87, $\beta=0.5$ ). Explicitly, the number of iterations of the rate-1/2 TTCM decoder is $\zeta=4$, the block size is $\eta=12,000$ symbols and an uncorrelated Rayleigh fading channel is considered.

The performance of our pre-coding based coded SPM scheme associated with CSI estimation errors are shown in Fig. 9. If there is CSI estimation error for the $R_{1} N\left(R_{2} N\right)$ and the DN link, the resultant $\rho_{1}$ in (7) and $\rho_{2}$ in (8) become:

$$
\begin{gathered}
\rho_{1}=\frac{\widetilde{h}_{R_{1} D}^{*}}{\left|\widetilde{h}_{R_{1} D}^{*}\right|^{2} \sqrt{G_{R_{1} D}}}, \\
\rho_{2}=\frac{\widetilde{h}_{R_{2} D}^{*}}{\left|\widetilde{h}_{R_{2} D}^{*}\right|^{2} \sqrt{G_{R_{2} D}}} .
\end{gathered}
$$

Hence, the pre-coding based twin-layer coded SPM-16QAM symbols received by the DN during the second TS is expressed as:

$$
y_{R D}=\frac{\alpha \widetilde{h}_{R_{1} D}^{*} h_{R_{1} D}}{\left|\widetilde{h}_{R_{1} D}^{*}\right|^{2}} x_{2}+\frac{\beta \widetilde{h}_{R_{2} D}^{*} h_{R_{2} D}}{\left|\widetilde{h}_{R_{2} D}^{*}\right|^{2}} x_{3}+n_{R D} .
$$

More specifically, we have:

$$
\begin{aligned}
& \widetilde{h}_{R_{1} D}=h_{R_{1} D}+\Delta_{1}, \\
& \widetilde{h}_{R_{2} D}=h_{R_{2} D}+\Delta_{2},
\end{aligned}
$$

where $\Delta_{1}$ is the CSI estimation error imposed on $h_{R_{1} D}$, and $\Delta_{2}$ is the CSI error contaminating $h_{R_{2} D}$. In order to simplify our discussions, we assume that both $\Delta_{1}$ and $\Delta_{2}$ obey the Gaussian distribution with zero mean and a variance of $\widetilde{\sigma}_{1}=\widetilde{\sigma}_{2}=\widetilde{\sigma}$. It can be seen from Fig. 9 that our pre-coding based twin-layer coded SPM-16QAM scheme is sensitive to the CSI estimation errors. Explicitly, an error floor around BER of $10^{-4}$ exists for the detection of $L_{2}$ and $L_{3}$ of the twin-layer SPM scheme when $\widetilde{\sigma}=0.001$. Upon increasing the CSI estimation error variance $\widetilde{\sigma}$, the performance of the pre-coding based coded SPM scheme will be dramatically reduced. The error floor will be eliminated at BER of $10^{-6}$ by employing sophisticated channel estimation 


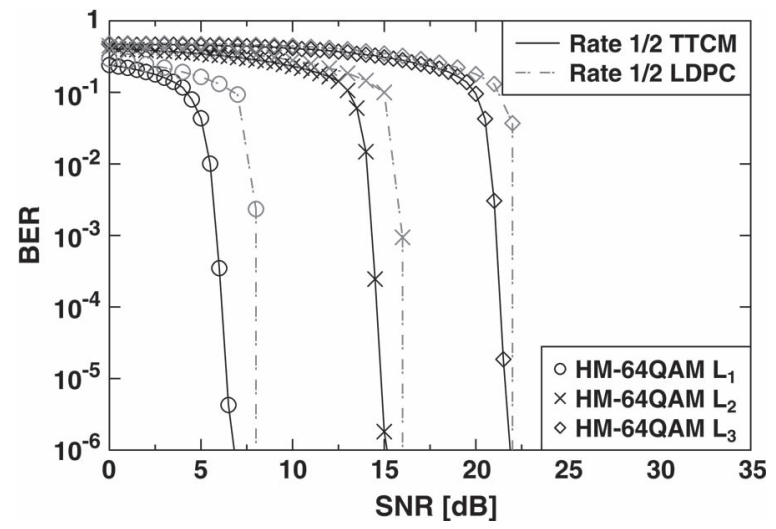

Fig. 10. The BER versus SNR performance of our triple-layer 64QAM TTCHM scheme and triple-layer 64QAM LDPC scheme. The triple-layer HM-64QAM ratio pair is $\left(R_{1}=1.5, R_{2}=0.8\right)$. The block size of the decoder of the two coding schemes is the same, which is $\eta=12,000$ symbols, the code-rate of the two coding schemes' encoder is $1 / 2$. For TTCM decoder, the iteration number is $\zeta=4$ and the maximum iteration number of LDPC decoder is $\zeta_{l}=20$. The system communicates over uncorrelated Rayleigh fading channel.

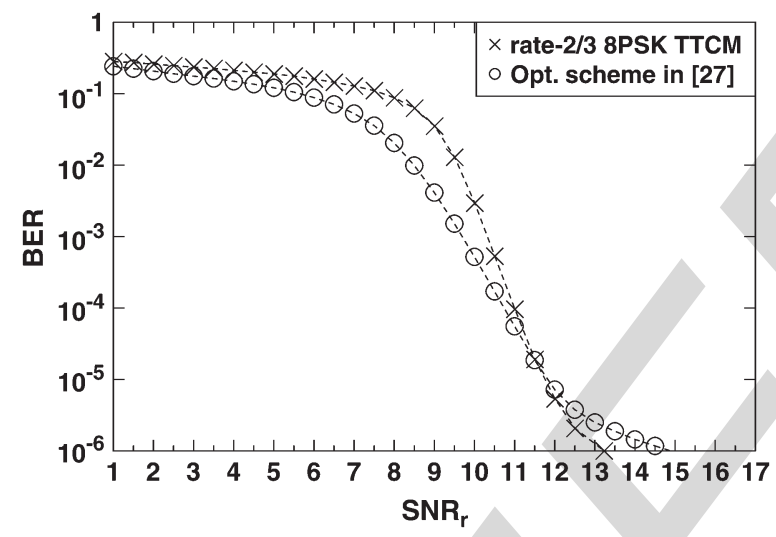

Fig. 11. The BER versus SNR performance of non-cooperative rate-2/3 8PSK TTCM and the optimized scheme in [26]. The number of iterations of the TTCM decoder in each of the three schemes is $\zeta=4$, the block size is $\eta=12,000$ symbols. An uncorrelated Rayleigh fading channel is considered.

schemes, which could reduce $\widetilde{\sigma}$ below a level of $10^{-5}$, as shown in Fig. 9.

\section{Comparisons With Other Systems}

Fig. 10 compares our rate-1/2 TTCM aided triple-layer HM-64QAM scheme to that of the same system but aided by a rate-1/2 LDPC code [38]. It can be observed that the bitbased LDPC coding scheme performs slightly worse than the symbol based TTCM coding scheme, even though the number of LDPC decoder iterations is much higher than that of the TTCM decoder. This is because a symbol-based scheme tends to have a lower convergence SNR than an equivalent bit-based scheme, as detailed in [39].

The BER performance curves of the non-cooperative rate- $2 / 3$ 8PSK TTCM and of our scheme in [26] are shown in Fig. 11. The throughputs of the two schemes are $2 \mathrm{bps}$ and $1.5 \mathrm{bps}$ (the scheme in [26] requires 2 TSs for cooperative transmission). Note that the throughput of the optimized scheme proposed in this paper is 1.455 bps. By contrast, the achievable throughput of the system proposed in this paper is $3 \times 0.97=2.91 \mathrm{bps}$, when the channel SNR is sufficiently high for the DN to detect all the three layers of the HM symbols in a single TS without the assistance of the RN. The $\mathrm{SNR}_{t}^{S N}$ required for achieving a BER of $10^{-6}$ for the non-cooperative rate-2/3 8PSK is $13.2 \mathrm{~dB}$, while that of the optimized scheme in [26] is $14.8 \mathrm{~dB}$. By contrast, the $\mathrm{SNR}_{t}^{S N}$ of the optimized scheme proposed in this paper is $6.81 \mathrm{~dB}$ and $\overline{S N R_{t}}$ is $6.94 \mathrm{~dB}$ per TS. Additionally, the simulation results of [13] show that in order to transmit a twin-layer HM-16QAM signal with the aid of two rate-1/2 H264/AVC encoders over an AWGN channel for achieving a BER of $10^{-6}$, their $\mathrm{SNR}_{t}^{S N}$ should be higher than $14 \mathrm{~dB}$, which is about $7.19 \mathrm{~dB}$ higher than that of our optimized scheme transmitting a triple-layer HM-64QAM signal over uncorrelated Rayleigh channels. It can be observed that the scheme optimized in this paper reduced the $\mathrm{SNR}_{t}^{S N}$ and $\overline{S N R_{t}}$ of the cooperative system.

\section{CONCLUSION}

In this paper, we proposed a TTCHM aided cooperative communication system. The system employed both pre-coding and SPM schemes at the two RNs, as detailed in Section II. The results have demonstrated that the best performance of the system is achieved at a HM-64QAM ratio pair of $\left(R_{1}=1.5\right.$, $\left.R_{2}=0.8\right)$ and the optimum SPM ratio pair is $(\alpha=0.87, \beta=$ 0.5 ). The optimized system requires an $\overline{S N R_{t}}$ of $6.94 \mathrm{~dB}$ per TS. It can be concluded that by employing HM in cooperative communications, both the $\operatorname{SNR}_{t}^{S N}$ may indeed be reduced, along with the $\overline{S N R_{t}}$ of the entire system. We note that spatial modulation [40] may also be employed for further reducing the transmit power dissipation of the entire system. The benefit of employing spatial modulation will be investigated in our future work.

\section{A. Derivation of (14)}

\section{APPENDIX}

Before normalization, the $L_{1}$ is represented as conventional square 4QAM symbols, hence we may have $d_{0}+d_{1}=\sqrt{2}$. Therefore, it can be expressed that:

$$
\delta_{1}=\frac{d_{0}}{2}=\frac{d_{0}}{\sqrt{2} \sqrt{2}}=\frac{d_{0}}{\sqrt{2}\left(d_{0}+d_{1}\right)}=\frac{1}{\sqrt{2}\left(1+R_{1}\right)} .
$$

\section{B. Derivation (15)}

As shown in Fig. 3, we have:

$$
R_{1}=\frac{d_{1}}{d_{0}} \quad \text { and } \quad R_{2}=\frac{d_{3}}{d_{2}} .
$$

Hence, we can write $d_{1}, d_{2}$ and $d_{3}$ as:

$$
\begin{aligned}
& d_{1}=d_{0} R_{1} \quad \text { and } \quad d_{3}=d_{2} R_{2}, \\
& d_{1}=d_{3}+2 \delta_{2} \quad \text { and } \quad d_{2}=d_{0}+2 \delta_{2},
\end{aligned}
$$


It may be observed from (56) and (57) that

$$
\delta_{2}=\frac{d_{1}-d_{3}}{2}=\frac{d_{0} R_{1}-d_{2} R_{2}}{2}=\frac{d_{0} R_{1}-\left(d_{0}+2 \delta_{2}\right) R_{2}}{2},
$$

where $\delta_{2}$ here may be expressed as:

$$
\delta_{2}=\frac{d_{0}\left(R_{1}-R_{2}\right)}{2\left(1+R_{2}\right)} .
$$

Upon substituting (54) into (59) we have:

$$
\delta_{2}=\frac{\left(R_{1}-R_{2}\right)}{\sqrt{2}\left(1+R_{1}\right)\left(1+R_{2}\right)} .
$$

\section{Restrictions on $R_{1}$}

It can be observe that:

$$
\begin{aligned}
& R_{1: \max }=\frac{d_{1_{\text {max }}}}{d_{0_{\text {min }}}}=\frac{\sqrt{2}}{0} \Rightarrow \infty, \\
& R_{1: \min }=\frac{d_{1_{\text {min }}}}{d_{0_{\max }}}=\frac{0}{\sqrt{2}} \Rightarrow 0,
\end{aligned}
$$

so we have $0<R_{1}<\infty$.

\section{Restrictions on $R_{2}$}

Note that $R_{2}$ is directly restricted by $R_{1}$ as follows:

$$
R_{2_{\max }}=\frac{d_{3_{\max }}}{d_{2_{\min }}}=\frac{d_{1}}{d_{0}} \Rightarrow R_{1} .
$$

If $R_{1}>1$, then $\max \left(\delta_{2}\right) \rightarrow d_{0} / 2$ and we have:

$$
R_{2_{\min }}=\frac{d_{3_{\min }}}{d_{2_{\max }}}=\frac{d_{1}-d_{0}}{2 d_{0}} \Rightarrow \frac{1}{2}\left(R_{1}-1\right) .
$$

By contrast, if $R_{1}<1$, then we have $\max \left(\delta_{2}\right) \rightarrow d_{3} / 2$ and hence:

$$
R_{2_{\min }}=\frac{d_{3_{\min }}}{d_{2_{\max }}}=\frac{0}{d_{0}+d_{3}} \Rightarrow 0 .
$$

\section{REFERENCES}

[1] C. Hellge, S. Mirta, T. Schierl, and T. Wiegand, "Mobile TV with SVC and hierarchical modulation for DVB-H broadcast services," in Proc. IEEE Int. Symp. BMSB, May 2009, pp. 1-5.

[2] S. Wang, S. Kwon, and B. K. Yi, "On enhancing hierarchical modulation," in Proc. IEEE Int. Symp. Broadband Multimedia Syst. Broadcast., Jun. 2008, pp. 1-6.

[3] R. Y. Kim and Y.-Y. Kim, "Symbol-level random network coded cooperation with hierarchical modulation in relay communication," IEEE Trans. Consum. Electron., vol. 55, no. 3, pp. 1280-1285, Oct. 2009.

[4] H. Jiang and P. A. Wilford, "A hierarchical modulation for upgrading digital broadcast systems," IEEE Trans. Broadcast., vol. 51, no. 2, pp. $223-$ 229, Jun. 2005.

[5] M. K. Chang and S. Y. Lee, "Performance analysis of cooperative communication system with hierarchical modulation over Rayleigh fading channel," IEEE Trans. Wireless Commun., vol. 8, no. 6, pp. 2848-2852, Jun. 2009.

[6] S. Y. Lee and K. C. Whang, "A collaborative cooperation scheme using hierarchical modulation," in Proc. IEEE 68th VTC, Sep. 2008, pp. 1-5.
[7] Z. Xuehua, A. Ghrayeb, and M. Hasna, "On hierarchical network coding versus opportunistic user selection for two-way relay channels with asymmetric data rates," IEEE Trans. Commun., vol. 61, no. 7, pp. 2900-2910, Jul. 2013.

[8] H. Mukhtar and M. EI-Tarhuni, "An adaptive hierarchical QAM scheme for enhanced bandwidth and power utilization," IEEE Trans. Commun., vol. 60, no. 8, pp. 2275-2284, Aug. 2012.

[9] K. Ramchandran, A. Ortega, K. M. Uz, and M. Vetterli, "Multiresolution broadcast for digital HDTV using joint source/channel coding," IEEE $J$. Sel. Areas Commun., vol. 11, no. 1, pp. 6-23, Jan. 1993.

[10] S. H. Chang, R. Minjoong, P. C. Cosman, and L. B. Mistein, "Optimized unequal error protection using multiplexed hierarchical modulation," IEEE Trans. Inf. Theory, vol. 58, no. 9, pp. 5816-5840, Sep. 2012.

[11] Y. J. Noli, H. C. Lee, and L. Y. Lee, "Design of unequal error protection for MIMO-OFDM systems with hierarchical signal constellations," J. Commun. Netw., vol. 9, no. 2, pp. 167-176, Jun. 2007.

[12] Y. C. Chang, S. W. Lee, and R. Komiya, "A low complexity hierarchical QAM symbol bits allocation algorithm for unequal error protection of wireless video transmission," IEEE Trans. Consum. Electron., vol. 55, no. 3, pp. 1089-1097, Aug. 2009.

[13] K. M. Alajel, W. Xiang, and Y. F. Wang, "Unequal error protection scheme based hierarchical 16-QAM for 3-D video transmission," IEEE Trans. Consum. Electron., vol. 58, no. 3, pp. 731-738, Aug. 2012.

[14] S. S. Arslan, P. C. Cosman, and L. B. Milstein, "Coded hierarchical modulation for wireless progressive image transmission," IEEE Trans. Veh. Technol., vol. 60, no. 9, pp. 4299-4313, Nov. 2011.

[15] S. S. Arslan, P. C. Cosman, and L. B. Milstein, "On hard decision upper bounds for coded M-ary hierarchical modulation," in Proc. 45th Аnnu. CISS, Mar. 2011, pp. 1-6.

[16] C. Hausl and J. Hagenauer, "Relay communication with hierarchical modulation," IEEE Commun. Lett., vol. 11, no. 1, pp. 64-66, Jan. 2007.

[17] L. Hanzo, T. H. Liew, B. L. Yeap, R. Y. S. Tee, and S. X. Ng, Turbo Coding, Turbo Equalisation and Space-Time Coding: EXIT-Chart-Aided Near-Capacity Designs for Wireless Channels, 2nd ed. Hoboken, NJ, USA: Wiley-IEEE Press, 2011

[18] C. H. Choi, Y. J. Kim, and G. H. Im, "Bit-interleaved coded transmission with multilevel modulation for non-orthogonal cooperative systems," IEEE Trans. Commun., vol. 59, no. 1, pp. 95-105, Jan. 2011.

[19] Z. Y. Li, M. Peng, and W. B. Wang, "Hierarchical modulated channel and network coding scheme in the multiple-access relay system," in Proc. IEEE 13th ICCT, Sep. 2011, pp. 984-988.

[20] J. M. Park, S. L. Kim, and J. H. Choi, "Hierarchically modulated network coding for asymmetric two-way relay systems," IEEE Trans. Veh. Technol., vol. 59, no. 5, pp. 2179-2184, Jun. 2010.

[21] Z. G. Du, P. L. Hong, K. P. Xue, and J. L. Peng, "Hierarchically modulated coded cooperation for relay system," in Proc. IEEE CCNC, Jan. 2012, pp. $812-816$.

[22] D. K. Kwon, W. J. Kim, K. H. Suh, and H. Lim, "A higher data-rate T-DMB system based on a hierarchical A-DPSK modulation," IEEE Trans. Broadcast., vol. 55, no. 1, pp. 42-50, Mar. 2009.

[23] P. Robertson and T. Worz, "Bandwidth-efficient turbo trellis-coded modulation using punctured component codes," IEEE J. Sel. Areas Commun., vol. 16, no. 2, pp. 206-218, Feb. 1998.

[24] S. X. Ng, J. Y. Chung, and L. Hanzo, "Turbo-detected unequal protection MPEG-4 wireless video telephony using multi-level coding, trellis coded modulation and space-time trellis coding," IEE Proc. Commun., vol. 152, no. 6, pp. 1116-1124, Dec. 2005.

[25] S. X. Ng, C. Y. Qian, D. D. Liang, and L. Hanzo, "Adaptive turbo trellis coded modulation aided distributed space-time trellis coding for cooperative communications," in Proc. IEEE 71st VTC-Spring, May 2010, pp. 1-5.

[26] H. Sun, Y. R. Shen, S. X. Ng, and L. Hanzo, "Turbo trellis coded hierarchical modulation for cooperative communications," in Proc. WCNC, Apr. 2013, pp. 2789-2794.

[27] S. H. Chang, R. Minijoong, P. C. Cosman, and L. B. Milstein, "Superposition MIMO coding for the broadcast of layered sources," IEEE Trans. Commun., vol. 59, no. 12, pp. 3240-3248, Dec. 2011.

[28] Y. K. Huo, M. El-Hajjar, and L. Hanzo, "Inter-layer FEC aided unequal error protection for multilayer video transmission in mobile TV," IEEE Trans. Circuits Syst. Video Technol., vol. 23, no. 9, pp. 1622-1634, Sep. 2013.

[29] H. Ochiai, P. Mitran, and V. Tarokh, "Design and analysis of collaborative diversity protocols for wireless sensor networks," in Proc. IEEE 60th VTC-Fall, Sep. 2004, vol. 7, pp. 4645-4649.

[30] C. Xu, S. X. Ng, and L. Hanzo, "Near-capacity irregular convolutional coded cooperative differential linear dispersion codes using 
multiplesymbol differential detection," IEEE Signal Process. Lett., vol. 18, no. 3, pp. 4645-4649, Mar. 2011.

[31] S. X. .Ng, Y. H. Li, and L. Hanzo, "Distributed turbo trellis coded modulation for cooperative communications," in Proc. ICC, Jun. 2009, pp. 1-5.

[32] A. Goldsmith, Wireless Communications, 1st ed. Cambridge, U.K.: Cambridge Univ. Press, 2005.

[33] S. M. Ross, Introduction to Probability Models, 9th ed. New York, NY, USA: Academic, 2007.

[34] H. Sun, S. X. Ng, and L. Hanzo, "Superposition coded modulation for cooperative communications," in Proc. IEEE VTC Fall, Sep. 2012, pp. $1-5$.

[35] A. Papoulis, Probability, Random Variables and Stochastic Processes, 4th ed. New York, NY, USA: McGraw-Hill, Jan. 2002.

[36] S. ten Brink, "Convergence behavior of iteratively decoded parallel concatenated codes," IEEE Trans. Commun., vol. 49, no. 10, pp. 1727-1737, Aug. 2002.

[37] S. X. Ng, J. Wang, and L. Hanzo, "Unveiling near-capacity code design: The realization of shannon's communication theory for MIMO channels," in Proc. IEEE ICC, May 2008, pp. 1415-1419.

[38] R. G. Gallager, "Low-density parity-check codes," IRE Trans. Inf. Theory, vol. 8, no. 1, pp. 21-28, Jan. 1962.

[39] B. Scanavino, G. Montorsi, and S. Benedetto, "Convergence properties of iterative decoders working at bit and symbol level," in Proc. IEEE GLOBECOM, Nov. 2001, vol. 2, pp. 1037-1041.

[40] M. D. Renzo, H. Haas, A. Ghrayeb, S. Sugiura, and L. Hanzo, "Spatial modulation for generalized MIMO: challenges, opportunities, implementation," Proc. IEEE, vol. 102, no. 1, pp. 56-103, Jan. 2014.

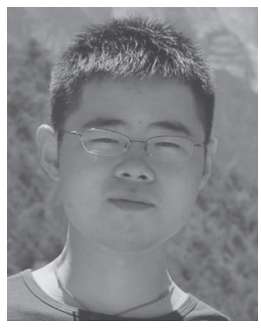

Hua Sun received the B.Eng. degree in electronics and information engineering from Huazhong University of Science and Technology, Wuhan, China, in 2009 and the M.Sc. degree (with distinction) in wireless communications from the University of Southampton, Southampton, U.K., in 2010. $\mathrm{He}$ is currently working toward the Ph.D. degree from the Research Group of Communications, Signal Processing and Control, School of Electronics and Computer Science, University of Southampton, Southampton, U.K. His research interests include superposition modulation, hierarchical modulation, turbo trellis-coded modulation, and cooperative communications.

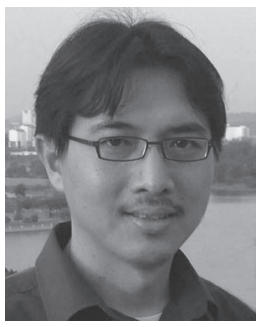

Soon Xin Ng (S'99-M'03-SM'08) received the B.Eng. degree (first class honor) in electronics engineering and the Ph.D. degree in wireless communications from the University of Southampton, Southampton, U.K., in 1999 and 2002, respectively. From 2003 to 2006, he was a Postdoctoral Research Fellow working on collaborative European research projects known as SCOUT, NEWCOM, and PHOENIX. Since August 2006, he has been with the School of Electronics and Computer Science, University of Southampton, where he is currently an Associate Professor. He is involved in the OPTIMIX and CONCERTO European projects, and the IU-ATC and UC4G projects. He is the author of over 180 papers and is the coauthor of two John Wiley/IEEE Press books in this field. His research interests include adaptive coded modulation, coded modulation, channel coding, space-time coding, joint source and channel coding, iterative detection, OFDM, MIMO, cooperative communications, distributed coding, quantum error correction codes and joint wireless-and-optical-fiber communications. He is a Chartered Engineer and a Fellow of the Higher Education Academy in the U.K.

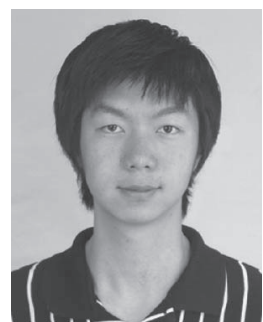

Chen Dong received the B.S. degree in electronic information sciences and technology from the University of Science and Technology of China, Hefei, China, in 2004; the M.Eng. degree in pattern recognition and automatic equipment from the University of Chinese Academy of Sciences, Beijing, China, in 2007; and the Ph.D. degree from the University of Southampton, Southampton, U.K. He is currently a Postdoctoral Researcher with the University of Southampton. His research interests include applied math, relay systems, channel modeling, and crosslayer optimization. He received a scholarship under the U.K.-China Scholarships for Excellence Programme and the Best Paper Award at the 2014 Fall IEEE Vehicular Technology Conference.

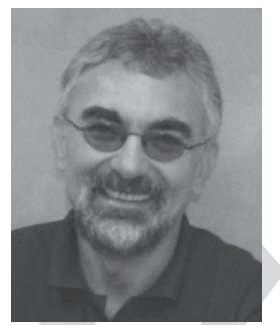

Lajos Hanzo (F'04) received the Master's degree in electronics, the Ph.D. degree, and the Doctor Honoris Causa degree from the Technical University of Budapest, Budapest, Hungary, in 1976, 1983, and 2009, respectively.

During his 38-year career in telecommunications, he has held various research and academic posts in Hungary, Germany, and U.K. Since 1986, he has been with the School of Electronics and Computer Science, University of Southampton, Southampton, U.K., where he is currently the Chair in telecommunications. He is also a Chaired Professor with Tsinghua University, Beijing, China. He has successfully supervised about $100 \mathrm{Ph}$.D. students, coauthored 20 John Wiley/IEEE Press books on mobile radio communications totalling in excess of 10000 pages, and published more than 1400 research entries at IEEE Xplore. Currently, he is directing a 100-strong academic research team, working on a range of research projects in the field of wireless multimedia communications sponsored by industry, the Engineering and Physical Sciences Research Council (EPSRC) U.K., the European Research Council through an Advanced Fellow Grant, and the Royal Society through the Wolfson Research Merit Award. He is an enthusiastic supporter of industrial and academic liaison, and he offers a range of industrial courses.

Dr. Hanzo has acted both as Technical Program Committee and General Chair of IEEE conferences, presented keynote lectures, and received a number of distinctions. He is also a Governor of the IEEE Vehicular Technology Society. From 2008 to 2012, he was the Editor-in-Chief of the IEEE Press. His research is funded by the European Research Council's Senior Research Fellow Grant. He is a Fellow of the Royal Academy of Engineering, Institution of Engineering and Technology, and European Association for Signal Processing. 